University of Navarra

\title{
A CAPACITATED COMMODITY TRADING MODEL WITH MARKET POWER
}

\author{
Victor Martínez-de-Albéniz \\ Josep Maria Vendrell Simón
}




\title{
A CAPACITATED COMMODITY TRADING MODEL WITH MARKET POWER
}

\author{
Victor Martínez-de-Albéniz' \\ Josep Maria Vendrell Simón²
}

\begin{abstract}
We consider in this paper the problem of a trader that may purchase a commodity in one market and resell it in another. The trader is capacitated: the trading volume is limited by operational constraints, e.g., logistics. The two markets quote different prices, but the spread is reduced when trading takes place. We are interested in finding the optimal trading policy across the markets so as to obtain the maximum profit in the long-term, taking into account that the trading activity influences the price processes, i.e., market power. Similarly to the no-market-power case, we find that the optimal policy is determined by three regions where (1) move as much as possible from one market to the other; (2) the same in the opposite direction; or (3) do nothing. We finally use the model to analyze the kerosene price differences between New York and Los Angeles.
\end{abstract}

\footnotetext{
${ }^{1}$ Professor, Production, Technology and Operations Management, IESE

${ }^{2}$ Assistant Research, IESE
} 


\section{A CAPACITATED COMMODITY TRADING MODEL WITH MARKET POWER}

\section{Introduction}

Commodities are the building block of a large part of our economy. Examples include not only minerals, metals and agricultural products such as iron ore, aluminum, silver, gold, sugar, coffee, rice, wheat, but also energy sources such as coal, oil, and natural or liquified gas, and even intermediary or manufactured products such as chemicals or generic drugs. They are nowadays relatively easily traded, can be physically delivered anywhere in the world, and sometimes can be stored for a reasonable period of time. The volume of commodity trading in the world is colossal: in 2006, more than 91 million 60-kg coffee bags were traded, more than 84 million barrels of oil are daily consumed, etc. Commodities are traded in very active markets, such as the Chicago Board of Trade (CBOT), the New York Mercantile Exchange (NYMEX), or the London Metal Exchange (LME), to cite a few. Prices are usually determined by market forces, rather than by the large suppliers or large buyers, with the exception perhaps of the Organization of the Petroleum Exporting Countries (OPEC). Spot prices at commodity markets exhibit several salient features: they are highly autocorrelated, and very volatile with rare but violent explosions in price. The volatility has been explained by inventory holding dynamics: where speculators carry inventory, the price remains stable, but when they are out-of-stock, the price fluctuates wildly, see Deaton and Laroque (1992). 
It is commonly assumed that the price of a commodity is the same in any point in the world, as occurs for stocks or bonds. This is not so, as the price in each location is adjusted for logistics costs and local market conditions (local balance of supply and demand) among others. For example, a coffee producer with a several factories in Europe will pay the different prices for deliveries in each one of the factories. One would assume that these differences are very small, or at least stable over time. As a matter of fact, the opposite is true, and price spreads, i.e., price difference between two locations, can be significant in the short term. As an example, which we analyze in detail in $\S 6.1$, we show in Figure 1 the evolution of kerosene jet-fuel spot prices, in New York and Los Angeles, over more than fifteen years. As can be seen in the left figure, the evolution of the price in these two locations is very similar. This implies that in the long-run the price spread is small. However, when examining, in the right figure, the price differences over the period, it is surprising to see short-run spreads of tens of cents of a dollar.
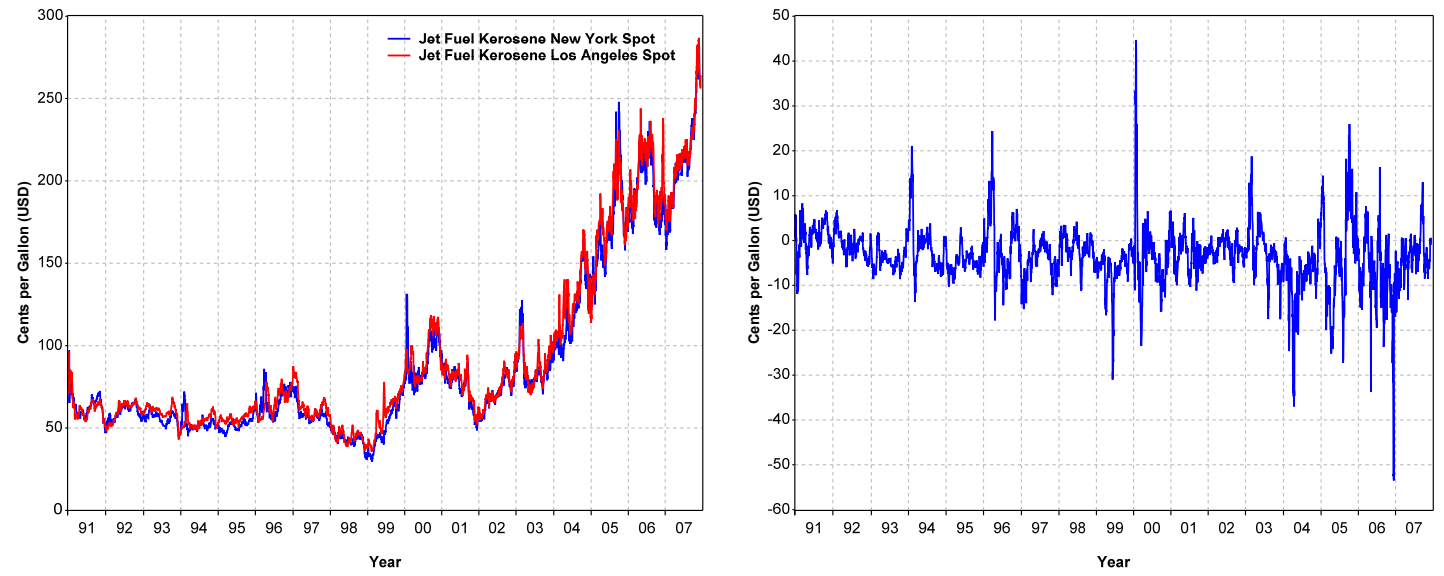

Figure 1: Plot of kerosene jet-fuel daily spot prices on the left (in USD cents per Gallon) in New York and Los Angeles, and the corresponding spread (price difference) $G_{t}=P_{t}^{N Y}-P_{t}^{L A}$.

In such market, there is a good opportunity for commodity traders to enter the market, and, in the short-run, make a profit. In stock and bond markets, these constitute arbitrage opportunities that disappear as soon as they appear. For commodities, however, it takes time for traders to close the price spreads to its average levels. In order to understand the market, it is important to elucidate how a trader should behave, according to its own trading capacity. Trading capacity may consist of shipping from one market to the other, which may be costly, for example. We must of course consider that the trading activity has an impact on the price levels in each market, i.e., the price at a location increases if a trader buys from it, and decreases if he sells to it: each trader has market power. 
In the current paper, we propose an analytical model of commodity trading in the presence of market power, which is new to the literature. We establish the rational behavior of traders between two markets and derive price spread dynamics based on the trading volumes. We consider two markets of commodities that quote different prices, and a trader is capable of purchasing in one market to resell in the other, within a trading capacity. For simplicity, we consider only two markets and assume that the inventory present between the two markets is constant, i.e., the amount stored is fixed (and is directly linked to the trading capacity). We first establish the structure of the optimal trading policy. This policy determines, given a realization of the current price spread between the two markets, how much (if any) volume must be bought/sold from/at the first market and sold/bought at/from the second market, so that the long-run average profit is maximized. When optimizing, the trader takes into account its impact on future spreads. We then describe the parameters of the optimal policy and we apply our model to kerosene prices in New York and Los Angeles, and illustrate the insights derived.

This model can be used for many different purposes. At an operational level, it allows the trader to specify when and how much to trade. At a more tactical and strategic level, the trader can predict the price spread distribution by looking at the trading capacity used for each spread level, and evaluate the impact of trading capacity additions.

The rest of the paper is organized as follows. In $\S 2$, we review the previous literature and how our paper is related to it. $\S 3$ describes in detail the model with its assumptions and notation. Then, in $\S 4$ we present the results for different market situations (monopoly, oligopoly), together with the optimality equations that characterize the optimal policy, and a numerical sensitivity analysis. $\S 5$ discusses several extensions of the basic model set-up. Finally, in $\S 6$ we show an application of our model to kerosene prices and discuss future research directions.

\section{Literature Review}

There is significant research on commodity prices and commodity trading, with at least two different literature streams: the first one, from economics and finance, and the second one, from operations management.

The first research stream studies the properties of commodity price evolution in established markets. One of the first important papers in this area is Deaton and Laroque (1992), which show that commodity prices have high autocorrelation and variability. They develop a rational expectations model for commodity prices that explains these features. They base their model on supply/demand forces and in particular on the existence of competitive speculators that hold 
inventory of the commodity, with which they expect to buy low and sell high. This behavior results in commodity prices normally staying within some limited range; here, speculators will build inventory when the price is low, and deplete it when it is high. As a result, the speculators act as regulators of the commodity prices. Sometimes, however, when supply and demand shocks push the price high, the speculators may run out of inventory, and hence spikes in prices will occur. Deaton and Laroque (1996) extend this work and find that speculation only is not enough to explain the high positive autocorrelation in observed commodity prices and that part of it is caused by the underlying processes of supply and demand.

Williams and Wright (1991), Chambers and Bailey (1996) and Routledge et al. (2000) also use a competitive rational expectations model to explain the properties of commodity prices in markets. They all consider the existence of convenience yield, which is defined in Hull (2003) as a measure of the benefits from owning a commodity asset versus holding a long futures contract on the asset. This is generally positive since carrying inventory allows the owner to make profits from trading opportunities that may arise, for instance from temporary local shortages; on the other hand, holding a futures contract does not allow it. For example, an oil refiner is unlikely to regard a futures contract on crude oil as equivalent to crude oil held in inventory: the crude oil in inventory can be an input to the refining process whereas a futures contract cannot be used for this purpose. The convenience yield typically reflects the market's expectations concerning the future availability of the commodity. As noted in Hull (2003), the greater the chances that shortages will occur, the higher the convenience yield. One can describe spot prices evolution by modeling convenience yields and spot prices as separate stochastic processes, possibly correlated, see Gibson and Schwartz (1990). Schwartz (1997) develops three variations of a mean-reverting stochastic model driven by one, two or three factors taking into account mean reversion of commodity prices, convenience yields and stochastic interest rates. Schwartz and Smith (2000) provide a two-factor stochastic spot price model determined by the combination of short-term and long-term factors, that allow volatility in both terms. This two-factor model is equivalent to the stochastic convenience yield model developed in Gibson and Schwartz (1990). These models are empirically validated for copper, gold and crude oil. Our paper does not directly model mean-reverting spot price processes or convenience yields, as in these papers, but focuses instead on modeling price spreads.

Routledge et al. (1998) consider the correlation between prices of different commodities within broad families (e.g., natural gas and electricity in energy). They model the substitutability of these commodities and find existence of equilibrium price processes, where rational agents convert one commodity into the other. In particular, they focus on natural gas and electricity, and analyze the spread (price difference) between these two commodities. This is known in the 
industry as the spark spread, and is used extensively by commodity traders. The industry also uses the dark spread, i.e., the spread between coal and electricity prices. In our paper, we focus on price spreads between different geographical locations, although the work could be extended to spreads between different commodities, as discussed in $\S 6.2$. We take a similar approach, and specifically consider the individual operational actions of rational traders, which allows us to describe in closed-form the agents' actions.

A second stream of literature on commodities exists in operations management. The papers in this group typically focus on the management of inventory of commodities, in the presence of price uncertainty, with buy/sell decisions in a single market. There is extensive literature on inventory management models, see for example Zipkin (2000). We refer readers to Goel and Gutierrez $(2004,2006,2007)$, and references therein for specific applications into commodities. These papers try to incorporate the information given by the convenience yield in the inventory and buy/sell decisions, e.g., Caldentey et al. (2007) for copper mining operations in Chile, where they use the stochastic process in Schwartz (1997) to model copper spot prices. In this sense, they try to combine finance and operations models.

In this group, Golabi (1985) models the prices of the commodity in future periods as random variables with known distribution functions. Assuming constant demand, he proves that a sequence of critical price levels at a given period determines the optimal ordering strategy. Wang (2001) proves that a myopic inventory policy is optimal for a multi-period model with stochastic demand and decreasing prices. Secomandi (2005) considers optimal commodity trading and provides a much more detailed view of the operations involved in trading. He focuses on storage assets, i.e. storage facilities or contracts that ensure that one will have the inventory at a pre-determined time. His model is based on inventory and well-behaved flow constraints. He shows that the optimal policy is, depending on the region, to buy and withdraw, to do nothing or to sell and inject. This type of policy is used for contract valuation in Secomandi (2004) and Wang et al. (2007).

Our work combines the ideas of price equilibrium, from economics and finance, together with a more detailed view of operations, as in Secomandi (2005). We are specifically interested in optimizing the inventory management policy, given that the trading activity may influence the price spread process. 


\section{The Model}

\subsection{Two Markets}

Consider the trading of a single commodity in two locations, A and B. Each one of these locations has its own local dynamics, and as a result the price at which the commodity trades in each place may be different. As mentioned in the introduction, for kerosene prices, these local price differences can be significant, see the descriptive statistics presented in $\S 6.1$.

Trading may also occur between $A$ and $B$. In particular, traders may choose to buy in one place and ship the commodity, at a cost, to the other. In reality, shipping of physical commodities cannot be done immediately, and is only feasible after a certain lead-time. For the sake of simplicity (and tractability), however, we assume that this lead-time is zero. Implicitly, we are assuming that at any given time, what is bought in one location is sold at the other. Thus, our basic model describes a pipeline, where no intermediate storage is allowed. In other words, a trader simply opens a faucet so that the commodity flows from A to B or vice-versa. We consider in addition that the pipeline capacity is given by the maximum achievable flow, in units per period. Note that the model could also handle a shipping process that does not involve a pipeline, as long as the inventory level in each part of the route remains constant. For instance, consider a set of trucks or ships that on each day can move towards A or towards B (all together). The quantity originating in A and arriving in B will be identical, and will be constrained by the maximum speed in the system. We denote by $q^{A B}$ the trading capacity from A to B, per period, and $q^{B A}$ the one from B to A.

In addition, we consider constant linear trading costs. We denote $c^{A B}$ the cost of moving one unit from $\mathrm{A}$ to $\mathrm{B}$, and $c^{B A}$ from $\mathrm{B}$ to $\mathrm{A}$. Using constant linear costs is a strong assumption, since it is independent of the price of the commodity ${ }^{1}$, but it has been made before in the literature, e.g., Goel and Gutierrez (2006).

Let $u_{t}^{A B}$ and $u_{t}^{B A}$ be the trading quantity from $\mathrm{A}$ to $\mathrm{B}$ and from $\mathrm{B}$ to $\mathrm{A}$ respectively, at time $t$. The capacity constraint implies that

$$
0 \leq u_{t}^{A B} \leq q^{A B} \text { and } 0 \leq u_{t}^{B A} \leq q^{B A}
$$

and the trading cost incurred is equal to $c^{A B} u_{t}^{A B}+c^{B A} u_{t}^{B A}$.

The trading policy, if decided rationally, should obviously depend on the prices quoted in A and B. For example, when $P_{t}^{B}-P_{t}^{A}>c^{A B}$, it is profitable to trade from A to B. Clearly, if the

\footnotetext{
${ }^{1}$ For instance, if the trading cost is a fraction of the dollar volume of trade, the cost would be a constant times the spot price at the buying market times the trading quantity. While this is numerically solvable, the analysis becomes intractable, see $\S 5.2$.
} 
current trading activity does not influence the future prices, the trader would try to maximize the present profit from trading at $t$, i.e.,

$$
\max _{u^{A B}, u^{B A} \geq 0}\left(P_{t}^{B}-P_{t}^{A}\right)\left(u_{t}^{A B}-u_{t}^{B A}\right)-c^{A B} u_{t}^{A B}-c^{B A} u_{t}^{B A} .
$$

It is easy to see that the optimal policy is such that either $u_{t}^{A B}=0$ or $u_{t}^{B A}=0$ and

$$
u_{t}:=u_{t}^{A B}-u_{t}^{B A}= \begin{cases}q^{A B} & \text { if } P_{t}^{B}-P_{t}^{A} \geq c^{A B} \\ 0 & \text { if } c^{A B} \geq P_{t}^{B}-P_{t}^{A} \geq-c^{B A} \\ -q^{B A} & \text { otherwise. }\end{cases}
$$

In other words, we will trade at maximum capacity provided that the price spread is bigger than the trading cost. As noted before, if $P_{t}^{A}$ and $P_{t}^{B}$ are independent of the trading quantities, this trading policy is optimal, as shown in Secomandi (2005).

Of course, if many traders follow this type of policy, the price at the cheaper market would rise and the price at the other market would go down, so the spread would tend to close over time. Hence, it is clear that the trading volume must impact the prices in the future. If this was not the case, we would see large spreads between locations, and more importantly, that these spreads may not close over time.

As a result, if the trading quantities do influence future prices, it is no longer clear what type of policy is now optimal for the trader. This requires modeling the relationship between global trading policies and the price spread process. This is done in the next section.

\subsection{The Price Processes}

We model time as a continuous variable, $t \geq 0$, and we denote the price of the commodity in markets $A$ and $B$ as $P_{t}^{A}$ and $P_{t}^{B}$ respectively. In the previous section, we have defined the net trading quantity at time $\mathrm{t}$ as $u_{t}:=u_{t}^{A B}-u_{t}^{B A}$. Using the same notation, $v_{t}:=v_{t}^{A B}-v_{t}^{B A}$ denotes what the other players in the market (competitors) trade from A to B. Of course, this is positive when the quantity flows from $\mathrm{A}$ to $\mathrm{B}$ and negative otherwise.

We model the the price processes at A and B as correlated random walk, i.e.,

$$
\left(\begin{array}{c}
d P_{t}^{A} \\
d P_{t}^{B}
\end{array}\right)=\left(\begin{array}{c}
\alpha\left(P_{t}^{A}, P_{t}^{B}\right)+\beta_{A}\left(u_{t}+v_{t}\right) \\
\alpha\left(P_{t}^{A}, P_{t}^{B}\right)-\beta_{B}\left(u_{t}+v_{t}\right)
\end{array}\right) d t+\left(\begin{array}{cc}
\sigma_{A 1} & \sigma_{A 2} \\
\sigma_{B 1} & \sigma_{B 2}
\end{array}\right)\left(\begin{array}{c}
d W_{t}^{1} \\
d W_{t}^{2}
\end{array}\right)
$$

where $W_{t}^{1}$ and $W_{t}^{2}$ are independent Wiener processes, $\alpha$ can be any exogenous function, and $\beta_{A}, \beta_{B}, \sigma \ldots \geq 0$.

As we can observe, the trade between $\mathrm{A}$ and $\mathrm{B}$ has a direct impact on the prices process, which captures the players' market power. Specifically, the total net trade from A to B at $t$, 


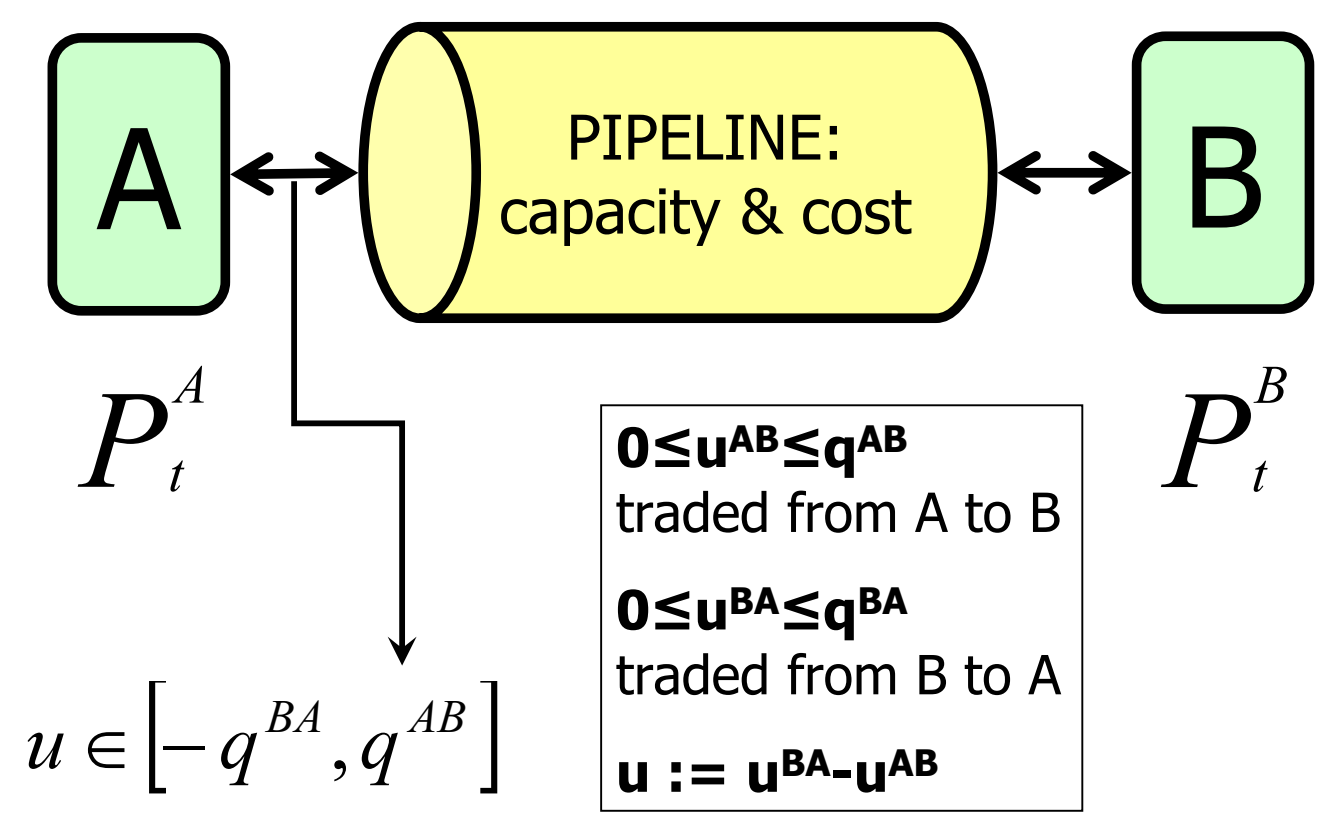

Figure 2: Summary of model features. The trader can ship $u^{A B}$ units of commodity from $\mathrm{A}$ to $\mathrm{B}$ and $u^{B A}$ units from B to A.

i.e., $u_{t}+v_{t}$, if positive, increases the price at $\mathrm{A}$ and decreases it at $\mathrm{B}$. This is qualitatively intuitive. Furthermore, we model this dependency as linear ${ }^{2}$.

Notice also that the proposed model could exhibit mean-reversion properties, as long as the mean-reverting adjustment is the same in both markets. For example, one could let $\alpha\left(P_{t}^{A}, P_{t}^{B}\right)=$ $\kappa\left[\mu-\left(\theta^{A} P_{t}^{A}+\theta^{B} P_{t}^{B}\right)\right]$, where $\mu$ would be the long-term mean price, defined as a weighted average of prices at $\mathrm{A}$ and $\mathrm{B}$.

Finally, in contrast with models in finance, e.g., Schwartz (1997), we consider linear random walks, instead of geometric ones. Our assumption guarantees tractability of the trading problem. However, since geometric Brownian motions might be more appropriate, we explore an alternative model in $\S 5.2$.

\footnotetext{
${ }^{2}$ This is a strong assumption, which is related to having linear price-quantity demand curves.
} 
Since the profit captured by the trader depends on the price spread, we define $G_{t}:=P_{t}^{B}-P_{t}^{A}$. From Equation (1), we have that $d G_{t}=-\left(\beta_{A}+\beta_{B}\right)\left(u_{t}+v_{t}\right) d t+\left(\sigma_{B 2}-\sigma_{A 2}\right) d W_{t}^{2}-\left(\sigma_{A 1}-\right.$ $\left.\sigma_{B 1}\right) d W_{t}^{1}$. Thus, $G_{t}$ is a stochastic process that can be expressed as

$$
d G_{t}=-\beta\left(u_{t}+v_{t}\right) d t+\sigma d W_{t}
$$

where $\beta=\beta_{A}+\beta_{B}, \sigma^{2}=\left(\sigma_{B 2}-\sigma_{A 2}\right)^{2}+\left(\sigma_{A 1}-\sigma_{B 1}\right)^{2}$ and $W_{t}$ a Wiener process.

In the remainder of the paper, we assume that the traded quantity $v_{t}$ (related to the rest of the market) only depends on $G_{t}$. This assumption guarantees tractability. As a result, since both revenue and cost depend only on $G_{t}$, it follows that, at optimality, the trader's policy $u_{t}$ should only be a function of the spread $G_{t} \cdot{ }^{3}$

Hence, considering that $u_{t}$ and $v_{t}$ are functions of $G_{t}$ only, i.e., $u_{t}=u\left(G_{t}\right)$ and $v_{t}=v\left(G_{t}\right)$, the random variable $G$, satisfies the stochastic differential equation $d G_{t}=A(G) d W_{t}+B(G) d t$, with $A(G)=\sigma$ and $B(G)=-\beta(u(G)+v(G))$. If for a certain $\epsilon>0, \lim _{G \rightarrow-\infty} B \geq \epsilon$ and $\lim _{G \rightarrow+\infty} B \leq-\epsilon, G$ has a stationary probability density function (p.d.f.), denoted $f(G)$, which satisfies

$$
\frac{1}{2} A^{2}(G) \frac{\partial^{2} f}{\partial G^{2}}-B(G) \frac{\partial f}{\partial G}=0 .
$$

This is known as the Kolmogorov equation, and is a backward parabolic partial differential

\footnotetext{
${ }^{3}$ Thus, in a game setting, this would be a Nash equilibrium, since the best-response to a trading policy that depends only on $G_{t}$ is a trading policy that depends only on $G_{t}$. This is true when each trader in the market has operating costs $c^{i j} u_{t}^{i j}$.
} 
equation $^{4}$. In our case, Equation (3) can be written as

$$
\frac{1}{2} \sigma^{2} \frac{\partial^{2} f}{\partial G^{2}}+\beta(u(G)+v(G)) \frac{\partial f}{\partial G}=0 .
$$

Thus, the control $u(G)$ has a direct influence on the p.d.f. of the spread. We denote it by $f_{u}(G)$ to make that dependency apparent. As an illustration, we show in Figure 3 the stationary p.d.f. for two controls $u$, where $v \equiv 0$.
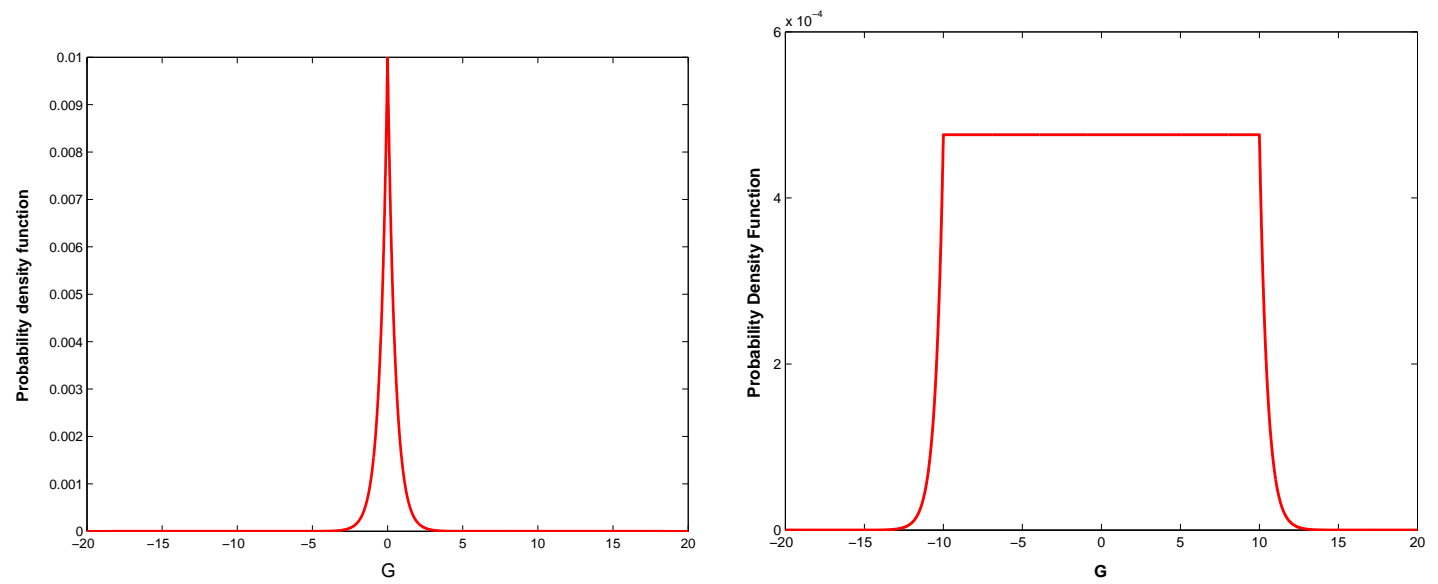

Figure 3: Stationary p.d.f. of $G$, with $v \equiv 0, \beta=1$ and $\sigma=1$, where, in the figure on the left, $u=-2$ for $G<0$ and $u=+2$ for $G \geq 0$; and, in the figure on the right, $u=-2$ for $G<-10, u=0$ for $-10 \leq G<10$ and $u=+2$ for $G \geq 10$.

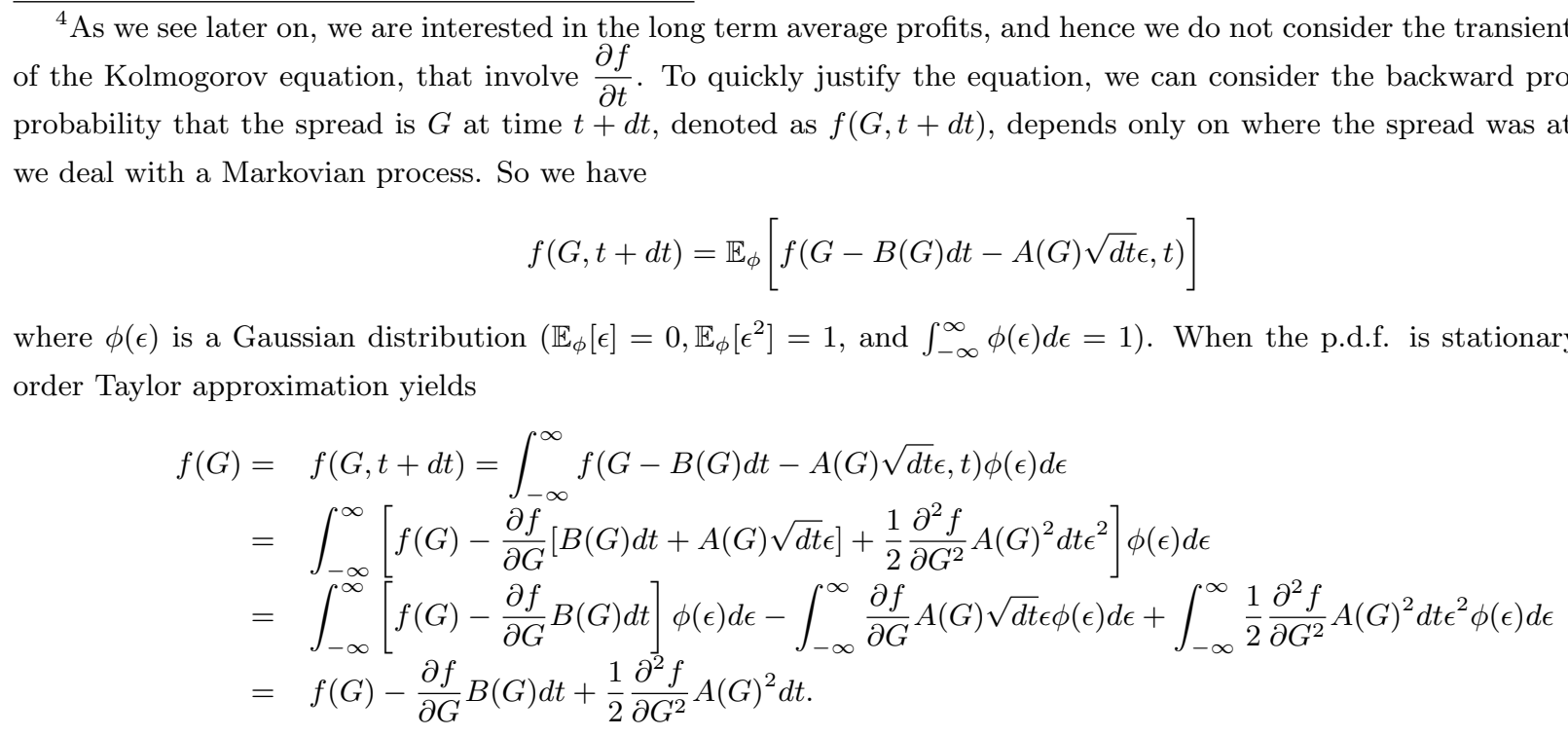

This yields Equation (3). For more references, see Wilmott (1997) for details on the methodology. 
For simplicity ${ }^{5}$, we restrict our attention to having both $u$ and $v$ piecewise constant functions. Now, if $u(G)+v(G)$ is piecewise constant, Equation (4) can be rewritten ${ }^{6}$ as

$$
u(G)=-\frac{\sigma^{2}}{2 \beta} \frac{f_{u}^{\prime}(G)}{f_{u}(G)}-v(G)
$$

This explicitly links the traded quantity $u$ to the price spread distribution $f_{u}$.

\subsection{Optimization of Trading Policies}

Given a realization of the price spread at time $t, G_{t}$, and given capacities $q^{A B}, q^{B A}$, we are interested in finding the optimal operating policy $u$ that maximizes the trader's long-run average profits. The optimal policy provides, given a price spread between the two markets, where and how much product to buy and sell. There are two components in the profit: the revenue, $G_{t} u_{t}$, and the trading cost, $c^{A B} u_{t}^{+}+c^{B A} u_{t}^{-}$, where $a^{+}=\max \{a, 0\}$ and $a^{-}=\max \{-a, 0\}$. In other words,

$$
J(u):=\mathbb{E}_{u}\left[G_{t} u_{t}-c^{A B} u_{t}^{+}-c^{B A} u_{t}^{-}\right]
$$

where the expectation is taken with respect to $f_{u}$, the stationary distribution of $G_{t}$. Hence, the problem in (6) can be equivalently expressed as

$$
\begin{aligned}
J^{*}=\sup _{u} J(u)= & \int_{-\infty}^{\infty}\left(G u(G)-c^{A B} u^{+}(G)-c^{B A} u^{-}(G)\right) f_{u}(G) d G \\
\text { subject to } & u(G)=-\frac{\sigma^{2}}{2 \beta} \frac{f_{u}^{\prime}(G)}{f_{u}(G)}-v(G) \\
& -q^{B A} \leq u(G) \leq q^{A B} \\
& f_{u} \text { a p.d.f. }
\end{aligned}
$$

where $f_{u}(G)$ is the spread distribution that depends on our control. This formulation has the advantage of being quite compact. Alternatively, we could have considered a discounted profit model, where the control would again depend only on $G_{t}$. However, by examining the alternative formulation,

$$
J\left(u, G_{0}\right)=\mathbb{E}\left[\int_{0}^{\infty}\left(G_{t} u\left(G_{t}\right)-c^{A B} u^{+}\left(G_{t}\right)-c^{B A} u^{-}\left(G_{t}\right)\right) e^{-r t} d t\right]
$$

one can observe that here we would need to consider the transient distribution of $G_{t}$, given that at time $t=0, G_{0}$ is the spread. A discrete version of the problem would raise similar

\footnotetext{
${ }^{5}$ The model can incorporate non-piecewise linear controls, but the analysis becomes significantly more complex.

${ }^{6}$ As $f(G)$ is a density function, the constant of integration must be 0 .
} 
complications:

$$
J\left(G_{0}\right)=\max _{-q^{B A} \leq u \leq q^{A B}}\left\{G_{0} u-c^{A B} u^{+}-c^{B A} u^{-}+\alpha \mathbb{E}_{\tilde{W}}\left[J\left(G_{0}-\beta\left(u+v\left(G_{0}\right)\right) \Delta t+\sigma \sqrt{\Delta t} \tilde{W}\right)\right]\right\}
$$

The problem posed by maximizing $J\left(u, G_{0}\right)$ in Equation (8) can be solved using optimal control. Interestingly, as we show in $\S 5.1$, the structure of the results that we derive for our basic problem setting, i.e., focusing on average profits, Equation (7), hold for this alternative formulation.

\section{Optimal Trading Policies}

After relating the trading quantity to the price spread distribution, we are interested in finding the optimal policy $u$ that maximizes the trader's problem (7). We first analyze the case of "monopoly", where there are no other traders between A and B (i.e., $v \equiv 0$ ) and then solve the general case.

\subsection{Monopoly: $v \equiv 0$}

We consider here an extreme case of our problem: the trader has no competitors trading between A and B. We call this a monopoly. Note that this does not mean that the trader is a price-setter in $\mathrm{A}$ or $\mathrm{B}$, but rather that he is the only player in the link between $\mathrm{A}$ and $\mathrm{B}$. In this case, Equation (5) can be written as $u(G)=-\frac{\sigma^{2}}{2 \beta} \frac{f^{\prime}(G)}{f(G)}$. Intuitively, one would think that this is the situation in which the trader can make highest profits. The next propositions show that, in fact, it is optimal to trade as little as possible, unless the price spread is really large, in order to maximize the average trading profit.

\section{Proposition 1 If}

(i) $c^{A B}=c^{B A}=0$ and

(ii) $v(G)=0 \forall G$,

then any policy is optimal in (7) and $J^{*}=\frac{\sigma^{2}}{2 \beta}$.

This result is somehow surprising. Indeed, in the short term, it would be better to instantly gain, at time $t, G_{t} q^{A B}$ if $G_{t}>0$ or $-G_{t} q^{B A}$ if $G_{t}<0$. However, by doing this every time, the trader would quickly modify the spread p.d.f. and push $G_{t}$ close to zero, which would reduce future profits. Alternatively, the trader could delay the trade until $\left|G_{t}\right|$ is large, in which case, he would obtain higher profits, but in fewer occasions. The proposition shows that both strategies will actually yield the same average profit in the long term when the trading cost is zero. In 
other words, the overall average benefit does not depend on the concrete feasible policy: it is the same to trade often, even with small spreads, or to delay the trade until spreads are large.

In contrast, the optimal policy does matter when there are trading costs.

\section{Proposition 2 If}

(i) $c^{A B}, c^{B A}>0$ and

(ii) $v(G)=0 \forall G$,

then the optimal policy for our trading problem (7) is the limit, when $M \longrightarrow \infty$, of

$$
u(G)=\left\{\begin{aligned}
-q^{B A} & \text { if } G \leq M \\
0 & \text { if }-M<G \leq M \\
q^{A B} & \text { if } G>M
\end{aligned}\right.
$$

and yields, at the limit, $J^{*}=\frac{\sigma^{2}}{2 \beta}$.

Proposition 2 incorporates cost. Proposition 1 implied that when $v \equiv 0$, and $c^{A B}=c^{B A}=0$, then the average long-run profit is independent of the trading quantity. When the costs are positive, the policy of not trading anything therefore yields the same average profit, and is optimal. Since such a policy is actually not feasible (the p.d.f. is not well-defined), we show that the same objective can be achieved as the limit of feasible policies.

\subsection{Oligopoly: $v(G) \neq 0$}

We have so far assumed that the trader was the only player with the ability to trade and make profits from the price spread. We consider below a market with several competitors. Competition might change the structure of the optimal policy because the distribution now also depends on how the competitors influence the market. We have denoted this influence as $v(G)$, where $v(G)$ is the total net quantity traded from A to B, by all the competitors. In that case, the control satisfies, from Equation (5),

$$
u(G)=-\frac{\sigma^{2}}{2 \beta} \frac{f^{\prime}(G)}{f(G)}-v(G) .
$$

When there are no trading costs, i.e., $c^{A B}=c^{B A}=0$, the objective function can be written as

$$
\begin{aligned}
\int_{-\infty}^{\infty} G u(G) f(G) d G & =\int_{-\infty}^{\infty} G\left(-\frac{\sigma^{2}}{2 \beta} \frac{f^{\prime}(G)}{f(G)}-v(G)\right) f(G) d G \\
& =\frac{\sigma^{2}}{2 \beta}+\int_{-\infty}^{\infty}-G v(G) f(G) d G
\end{aligned}
$$


after integrating by parts $G f^{\prime}(G)$. Hence, the maximization problem is equivalent

$$
\begin{aligned}
\sup _{f} & \int_{-\infty}^{\infty}-G v(G) f(G) d G \\
\text { subject to } & -q^{B A} \leq-\frac{\sigma^{2}}{2 \beta} \frac{f^{\prime}(G)}{f(G)}-v(G) \leq q^{A B} \\
& f \text { a p.d.f. }
\end{aligned}
$$

This reformulation has the advantage that the trading volume $u$ is no longer present. We can thus optimize directly taking $f$ as the decision variable. The optimization problem can be fully solved, with the support of a technical lemma (see appendix). In short, we find necessary conditions that characterize the optimal function $f$. Since the p.d.f. and the trading quantity $u(G)$ are directly related, this is equivalent to finding the optimal trading policy.

\section{Proposition 3 If}

(i) $c^{A B}=c^{B A}=0$ and

(ii) $v(G)$ is non-decreasing, $\lim _{G \rightarrow+\infty} v(G)>0$ and $\lim _{G \rightarrow-\infty} v(G)<0$,

then there exists $m$ such that the optimal policy for our trading problem (7) is

$$
u(G)=\left\{\begin{aligned}
-q^{B A} & \text { if } G \leq m \\
q^{A B} & \text { if } G>m .
\end{aligned}\right.
$$

The proposition requires a regularity assumption on $v$, in order to have a well-defined optimal policy, and avoid asymptotically optimal solutions, as in Proposition 2. Under such condition, when there are no costs, we find that the optimal trading policy is to trade as much as possible from one market to the other, with the trading direction depending on whether the spread $G$ is higher or lower that a threshold $m$. We show the result by establishing that, in problem (10), one of the two constraints on $\frac{f^{\prime}}{f}$ is always binding.

We next generalize the preceding results to the general case, with $c^{A B}, c^{B A}>0$. In that case, similarly to Equation (10), the trading problem (7) is equivalent to

$$
\begin{aligned}
\sup _{f} & \int_{-\infty}^{\infty}\left[-G v(G)-c^{B A}\left(-\frac{\sigma^{2}}{2 \beta} \frac{f^{\prime}(G)}{f(G)}-v(G)\right)^{-}-c^{A B}\left(-\frac{\sigma^{2}}{2 \beta} \frac{f^{\prime}(G)}{f(G)}-v(G)\right)^{+}\right] f(G) d G \\
\text { subject to } & -q^{B A} \leq-\frac{\sigma^{2}}{2 \beta} \frac{f^{\prime}(G)}{f(G)}-v(G) \leq q^{A B} \\
& f \text { a p.d.f. }
\end{aligned}
$$

Solving the problem above yields the following theorem. 


\section{Theorem 1 If}

(i) $c^{A B}, c^{B A}>0$ and

(ii) $v(G)$ is non-decreasing, $\lim _{G \rightarrow+\infty} v(G)>0$ and $\lim _{G \rightarrow-\infty} v(G)<0$,

then there exists $m^{B A}, m^{A B}$, such that $m^{B A} \leq m^{A B}$, and the optimal policy for our trading problem (7) is

$$
u(G)=\left\{\begin{aligned}
-q^{B A} & \text { if } G \leq m^{B A} \\
0 & \text { if } m^{B A}<G \leq m^{A B} \\
q^{A B} & \text { if } G>m^{A B} .
\end{aligned}\right.
$$

In essence, the optimal trading policy is determined by three regions: $\left[-\infty, m^{B A}\right]$ where it is optimal to move as much product as possible from market $\mathrm{B}$ to the market $\mathrm{A} ;\left(m^{A B}, \infty\right]$, where the same is true from $\mathrm{A}$ to $\mathrm{B}$, the opposite direction; and $\left(m^{B A}, m^{A B}\right]$ where it is optimal to do nothing. This is the same structure as in the case with no market power. In our notation, with no market power, $m^{B A}=-c^{B A}$ and $m^{A B}=c_{A B}$. Hence, market power changes the optimal trading policy by delaying the point where the traders begin to ship product from one market to the other.

The theorem thus generalizes Propositions 1, 2 and 3. The proof of the theorem is interesting, as it uses necessary conditions to determine the thresholds $m^{B A}, m^{A B}$. These conditions are analyzed in more detail in the next section.

\subsection{The Optimal Trading Thresholds}

In this section, we establish how to compute the optimal trading policy, or equivalently, the spread p.d.f. We show as well some properties of the problem depending on the function $v(G)$.

Interestingly, the optimal policy derived in Theorem 1 is given by three optimality equations. Knowing that the optimal policy is given by two thresholds $m^{B A}, m^{A B}$, since

$$
-\frac{\sigma^{2}}{2 \beta} \frac{f^{\prime}(G)}{f(G)}= \begin{cases}-q^{B A}+v(G) & \text { if } G \leq m^{B A} \\ v(G) & \text { if } m^{B A}<G \leq m^{A B} \\ q^{A B}+v(G) & \text { if } G>m^{A B}\end{cases}
$$

let

$$
\varphi(G)= \begin{cases}e^{\int_{m}^{G} B A\left(-v(t)+q^{B A}\right) \frac{2 \beta}{\sigma^{2}} d t} & \text { if } G \leq m^{B A} \\ e^{\int_{m}^{G} B A-v(t) \frac{2 \beta}{\sigma^{2}} d t} & \text { if } m^{B A}<G \leq m^{A B} \\ e^{\int_{m}^{m^{B} A B}-v(t) \frac{2 \beta}{\sigma^{2}} d t} e^{\int_{m}^{G} A B\left(-v(t)-q^{A B}\right) \frac{2 \beta}{\sigma^{2}} d t} & \text { if } G>m^{A B} .\end{cases}
$$


Hence, we have that $f=\frac{\varphi}{\int_{-\infty}^{\infty} \varphi}$. We can express the objective function as

$$
W:=\left(\frac{1}{\int_{-\infty}^{\infty} \varphi d G}\right)\left(\begin{array}{c}
\int_{-\infty}^{m^{B A}}\left(-G v(G)-c^{B A} q^{B A}\right) \varphi(G) d G \\
+\int_{m^{B A}}^{m^{A B}}-G v(G) \varphi(G) d G \\
+\int_{m^{A B}}^{\infty}\left(-G v(G)-c^{A B} q^{A B}\right) \varphi(G) d G
\end{array}\right) .
$$

Maximizing $W$ with respect to $m^{B A}$ and $m^{A B}$ yields the following optimality conditions.

Proposition 4 Consider $u$ be the optimal control as defined in Theorem (1). $m^{B A}, m^{A B}$ and $W$ are jointly characterized by Equation (13) together with

$$
\begin{aligned}
& W=\frac{\int_{m^{A B}}^{\infty}-\left(G-c^{A B}\right) v(G) \varphi(G) d G}{\int_{m^{A B}}^{\infty} \varphi}, \\
& W=\frac{\int_{-\infty}^{m^{B A}}-\left(G+c^{B A}\right) v(G) \varphi(G) d G}{\int_{-\infty}^{m^{B A}} \varphi} .
\end{aligned}
$$

With the equations above, we can find numerically the value of the parameters and hence the optimal function $f(G)$. In addition, under some properties of $v(G)$, the equations allow us to derive some properties on the optimal thresholds.

Proposition 5 If $c^{A B}=c^{B A}, q^{A B}=q^{B A}$ and $v(G)$ is anti-symmetrical, i.e., $v(-G)=-v(G)$ for all $G$, then $m^{B A}=-m^{A B}$.

Furthermore, for the extreme case where the trader's capacity is very small (very limited market power) $m^{B A}$ and $m^{A B}$ are closer to the trading costs.

Proposition 6 If $q^{A B}, q^{B A} \rightarrow 0$, then $m^{B A} \rightarrow-c^{B A}$ and $m^{A B} \rightarrow c^{A B}$.

This result shows that our model is robust and that in the limit (when $q^{A B}=q^{B A}=0$ ), our optimal policy is the same as that of a rational trader with no market power. In addition, one is able to obtain first-order approximations of $m^{B A}$ and $m^{A B}$ when the capacities $q^{A B}, q^{B A}$ are small, see Appendix.

Proposition 6 allows us to derive some interesting conclusions in a "perfect" market, where all traders are small. In that situation, each trader is going to start trading when the spread is beyond its transaction cost. As a result, there is a direct relationship between traders' costs, installed capacities and the market spread long-run distribution. We use this assumption later 
on, in $\S 6.1$, in order to estimate the amount of capacity present in the market based on the price spread distribution.

\subsection{Sensitivity analysis}

In this section we show some properties of the model with respect to small changes in the parameters. We have done so numerically with MATLAB because the analytical expressions for the partial derivatives are untractable. For this purpose, we have taken two different approaches. We have implemented the explicit analytical function defined in $\S 4.3$ and compared the results with explicit simulations of concrete policies. The results found in both cases are identical so our model is robust. Figure 4 is an example of this: the simulated p.d.f. (with 10,000 data points) converges to the analytical p.d.f. Since both approaches yield similar results, we have used below the analytical implementation because the computations are faster than with simulations.

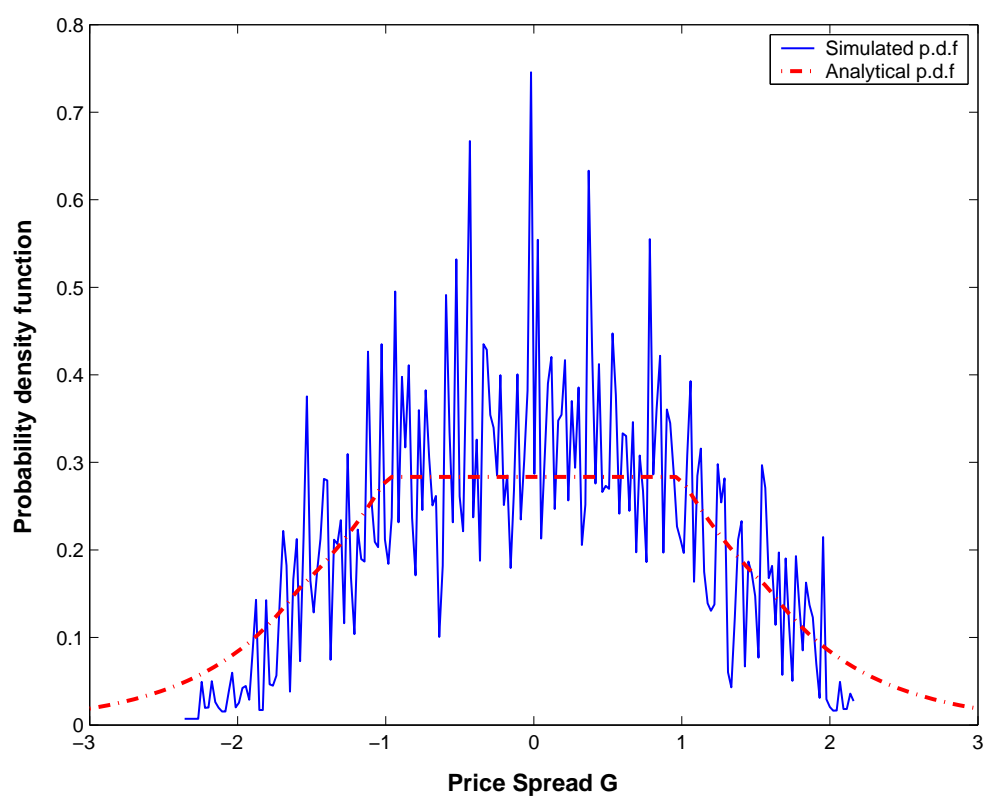

Figure 4: Comparison between the analytical pdf (red) in blue and the simulated pdf (blue).

For given $c^{B A}, c^{A B}, q^{B A}, q^{A B}, \beta, \sigma, v(\cdot)$, the optimal thresholds $m^{B A}, m^{A B}$ are found, through an exhaustive search, even though it would be computationally more efficient just to solve Equations (13), (14) and (15). We also compute the average profit $J^{*}$ defined in (6).

Without lost of generality we have assumed that $\frac{\sigma^{2}}{2 \beta}=1$. This is a scaling assumption. In addition, we have considered two main cases: 
- the symmetric trader: $c^{B A}=c^{B A}=c$ and $q^{B A}=q^{A B}=q$;

- the uni-directional trader: $c^{B A}=\infty, c^{A B}=c$ and $q^{B A}=0, q^{A B}=q$.

For both scenarios, we numerically show the impact of $c$ and $q$ on the optimal average profit $J^{*}=\frac{\sigma^{2}}{2 \beta}-W$, and on the thresholds $m^{B A}, m^{A B}$. Note that for the symmetric trader scenario, we can apply Proposition 5 and hence $m^{B A}=-m^{A B}$.
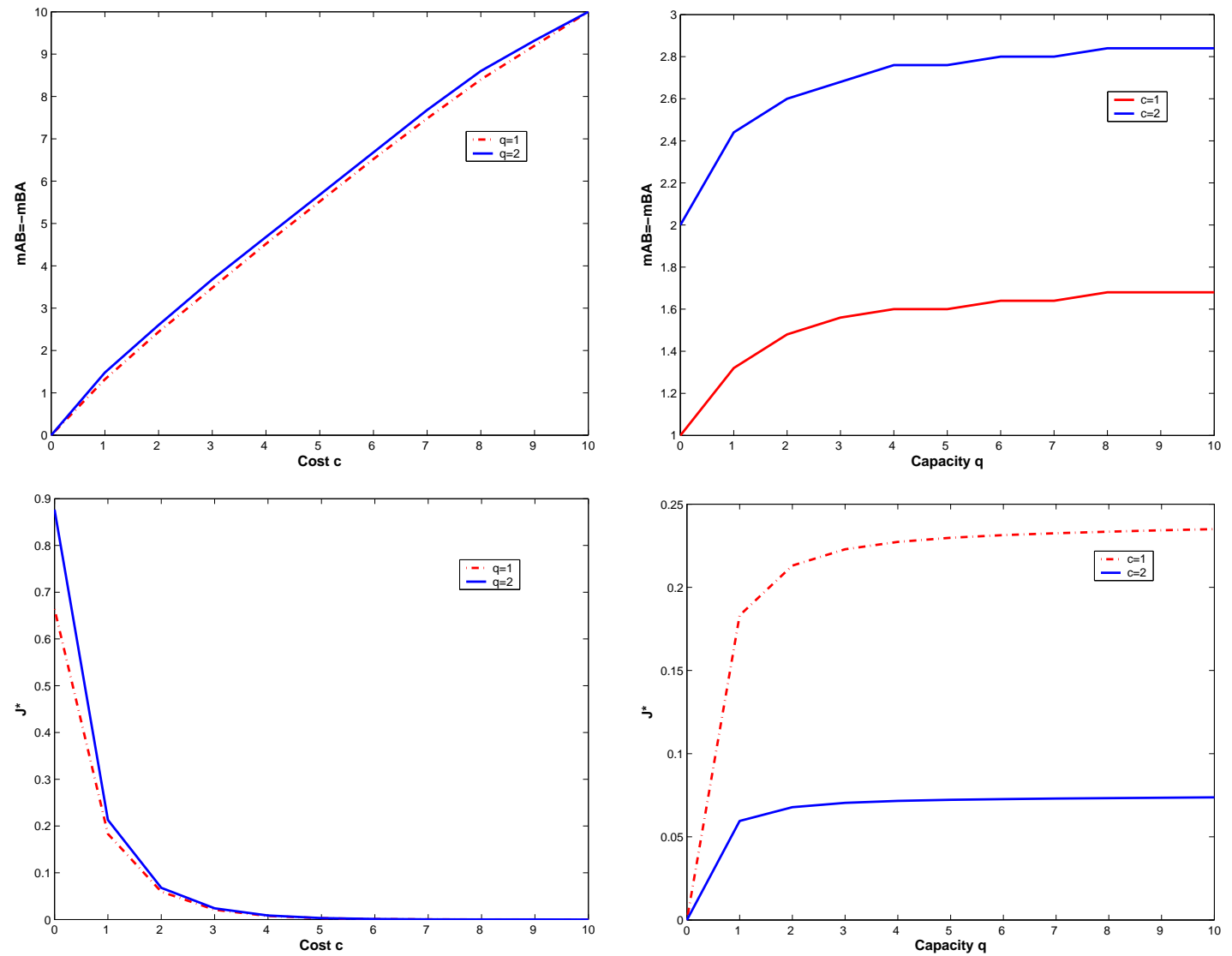

Figure 5: The symmetric trader. The top figures depict the changes of the optimal $m^{B A}, m^{A B}$ with changes in $c$ (left) and $q$ (right). The bottom figures show the corresponding changes in average profit $J^{*}$, again with changes in $c$ (left) and $q$ (right). In these figures, we consider that $v(G)=1$ when $G \geq 1$, $v(G)=-1$ when $G \leq-1$ and $v(G)=0$ otherwise.

We observe in Figures 5 and 6 that, as one would expect, the trader's average profit decreases to zero with cost. Any capacity is actually useless for $c \geq 5$, since, as the competitor starts trading $|v|=1$ for $\left|G_{t}\right| \geq 1$, the spread never goes above $c$. The threshold at which the trader begins to operate is actually very close to the cost, for the entire range.

In addition, the trader's profit is concave in the capacity, implying decreasing marginal 

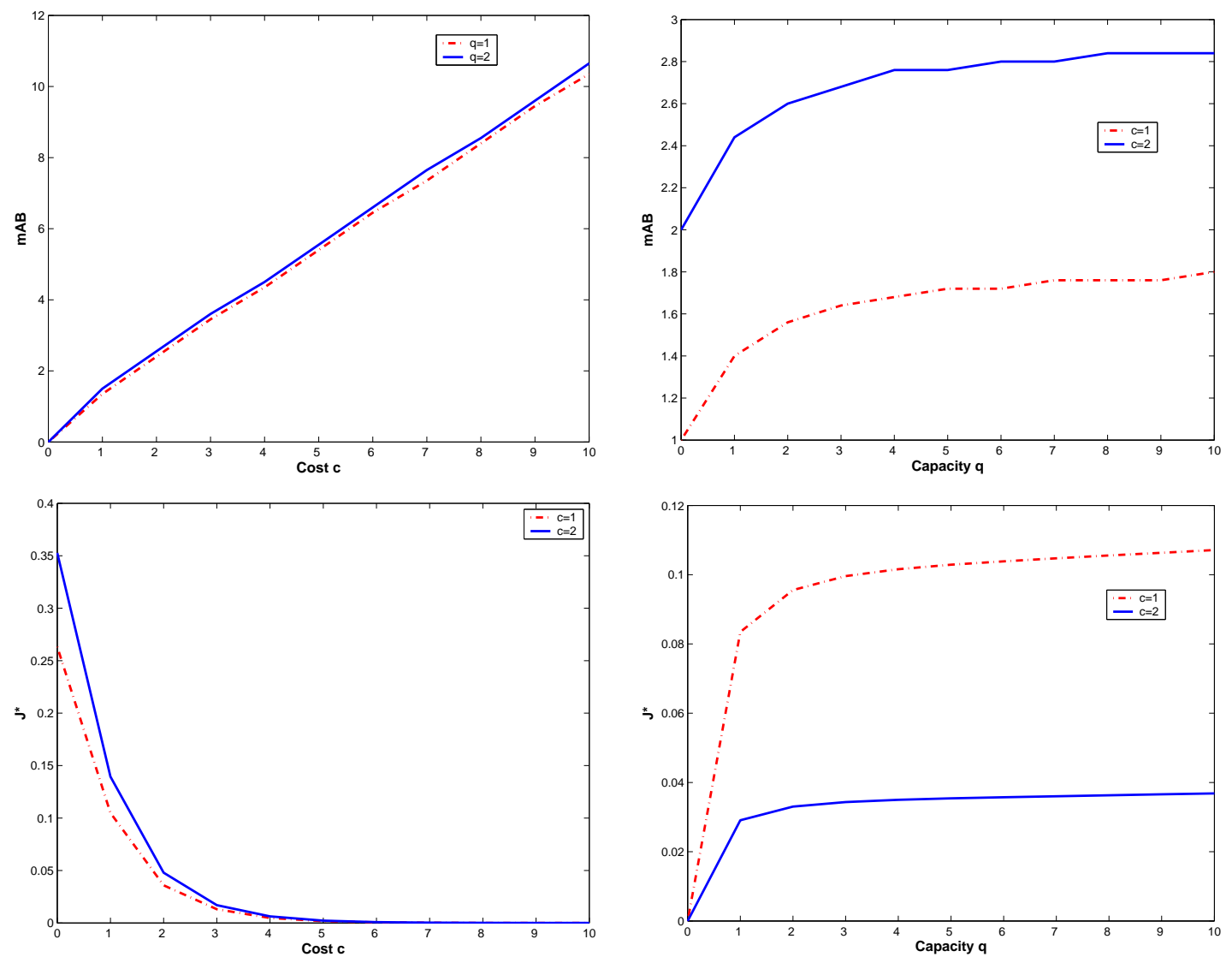

Figure 6: The uni-directional trader. The top figures depict the changes of the optimal $m^{A B}$ with changes in $c$ (left) and $q$ (right). The bottom figures show the corresponding changes in average profit $J^{*}$, again with changes in $c$ (left) and $q$ (right). In these figures, we consider that $v(G)=1$ when $G \geq 1$, $v(G)=-1$ when $G \leq-1$ and $v(G)=0$ otherwise. 
returns on the capacity. The marginal value of capacity is very small for $q \geq 2$, since more capacity tends to reduce the price spreads. As the capacity grows, the threshold also increases, and can be quite high compared to the cost, e.g., for the symmetric trader, when $q=5, c=1$ and $m^{A B} \approx 1.6$.

Interestingly, comparing Figures 5 and 6, one can see that the symmetric trader makes slightly more than twice the profit for the unidirectional trader. The optimal trading thresholds are very similar in both cases. This suggests that, in the case of a symmetric trader, the thresholds could be computed independently, i.e., find $m^{A B}$ using $c^{A B}, q^{A B}>0$ and $q^{B A}=0$, and similarly for $m^{B A}$. This policy would be close to optimal.

\section{Extensions}

\subsection{Discounted Profit Maximization}

One of the strong assumptions of our model is that the trader cares about long-term average profit. This assumption does simplify the analysis, as it allows treating the objective in an explicit way, and, as a result, computing closed-form thresholds for the optimal policy. In this section, we complement the analysis with the discounted profit maximization case. We use optimal control theory to solve the problem. Denoting as $J\left(G_{0}\right)$ the profit-to-go, function of $G_{0}$, the current price spread, the maximization problem can be written as

$$
\max _{-q^{B A} \leq u_{t} \leq q^{A B}} J\left(G_{0}\right)=\mathbb{E}\left\{\int_{0}^{\infty} e^{-r t}\left(G_{t} u_{t}-c^{B A} u_{t}^{-}-c^{A B} u_{t}^{+}\right) d t\right\}
$$

where $r$ is the instantaneous discount factor, and such that $d G_{t}=-\beta\left(u_{t}+v\left(G_{t}\right)\right) d t+\sigma d W_{t}$

The optimality conditions, see Bertsekas [1], can be captured through the Hamilton-JacobiBellman (HJB) equation. First, the optimal control $u^{*}(G)$ is given by

$$
\max _{-q^{B A} \leq u \leq q^{A B}}\left(G u-c^{B A} u^{-}-c^{A B} u^{+}\right)-\beta \frac{d J}{d G} u .
$$

This implies that $u^{*}(G)=-q^{B A}, 0, q^{A B}$. Hence, a bang-bang policy is optimal in this case as well. In addition, if $G-\beta \frac{d J}{d G}$ is increasing when equal to $-c^{B A}, 0, c^{A B}$, then there exists $m^{B A}, m^{A B}$ such that $u^{*}(G)=-q^{B A}$ when $G \leq m^{B A} ; u^{*}(G)=q^{A B}$ when $G \geq m^{A B}$; and finally, $u^{*}(G)=0$ when $m^{B A} \leq G \leq m^{A B} 7$.

\footnotetext{
${ }^{7}$ In that case, the HJB equation satisfies, when $G \leq m^{B A}, \frac{\sigma^{2}}{2} \frac{d^{2} J}{d G^{2}}-\beta\left(-q^{B A}+v\right) \frac{d J}{d G}-r J-\left(G-c^{B A}\right) q^{B A}=0$; when $G \geq m^{A B}, \frac{\sigma^{2}}{2} \frac{d^{2} J}{d G^{2}}-\beta\left(q^{A B}+v\right) \frac{d J}{d G}-r J+\left(G-c^{A B}\right) q^{A B}=0 ;$ and when $m^{B A} \leq G \leq m^{A B}, \frac{\sigma^{2}}{2} \frac{d^{2} J}{d G^{2}}-\beta v \frac{d J}{d G}-r J=0$.
} 


\subsection{Alternative Price Processes, Cost Structures and Competitor Actions}

One of the main advantages in our model is to be able to recast a problem with two stochastic processes, $P_{t}^{A}$ and $P_{t}^{B}$, into a problem with a single stochastic process $G_{t}=P_{t}^{B}-P_{t}^{A}$. This can be done under three main assumptions:

- that $d G_{t}$ can be expressed only as a function of $G_{t}$ (for instance, that the actions of the competitors $v_{t}$ only depend on $G_{t}$, not on $\left.\left(P_{t}^{A}, P_{t}^{B}\right)\right)$;

- and that the cost function of the trader is linear, which implies that it is independent of $\left(P_{t}^{A}, P_{t}^{B}\right)$.

When any of these two assumptions is not satisfied, then the approach developed in the paper is significantly more complicated. We provide guidelines on how to deal with these more general models. The generic formulation for the price processes, replacing Equation (1), is:

$$
\begin{aligned}
& d P_{t}^{A}=\left[\alpha^{A}\left(P_{t}^{A}, P_{t}^{B}\right)+\beta^{A}\left(P_{t}^{A}, P_{t}^{B}\right)\left(u_{t}+v_{t}\left(P_{t}^{A}, P_{t}^{B}\right)\right)\right] d t+\sigma^{A}\left(P_{t}^{A}, P_{t}^{B}\right) d W_{t}^{A} \\
& d P_{t}^{B}=\left[\alpha^{B}\left(P_{t}^{A}, P_{t}^{B}\right)-\beta^{B}\left(P_{t}^{A}, P_{t}^{B}\right)\left(u_{t}+v_{t}\left(P_{t}^{A}, P_{t}^{B}\right)\right)\right] d t+\sigma^{B}\left(P_{t}^{A}, P_{t}^{B}\right) d W_{t}^{B}
\end{aligned}
$$

One particular case of these is the geometric Brownian motion, where $\alpha^{i}=0, \beta^{i}=P_{t}^{i} \bar{\beta}^{i}$, and $\sigma^{i}=P_{t}^{i} \bar{\sigma}^{i}$, with $\bar{\beta}^{i}, \bar{\sigma}^{i}$ constants.

The objective function thus becomes

$$
\begin{aligned}
J(u) & =\max _{u} \mathbb{E}\left\{\left(P_{t}^{B}-P_{t}^{A}\right) u-c\left(P_{t}^{A}, P_{t}^{B}, u\right)\right\} \\
& =\max _{u} \iint_{-\infty}^{+\infty}\left[\left(p^{B}-p^{A}\right) u\left(p^{A}, p^{B}\right)-c\left(p^{A}, p^{B}, u\left(p^{A}, p^{B}\right)\right)\right] f_{u}\left(p^{A}, p^{B}\right) d p^{A} d p^{B},
\end{aligned}
$$

where $f_{u}$ satisfies a partial differential equation similar to (3), but in two variables. In this case, no closed form solution can be easily computed, and $f_{u}$ should be found numerically.

\subsection{Trading in a Network}

The model developed in this paper considers trading between two pre-determined locations. However, in reality trading opportunities are possible between many potential origins and destinations. For this purpose, in a network of $n$ points (a connected graph), one can define the trading quantities between $i$ and $j$ as $u^{i j}$, the existing trading capacity as $q^{i j}$ and the trading unit $\operatorname{cost} c^{i j}$. We must also redefine the price process evolution from Equation (1) as

$$
d P_{t}^{i}=\left[\alpha\left(\mathbf{P}_{t}\right)+\sum_{j \neq i} \beta^{i j}\left(u_{t}^{i j}+v_{t}^{i j}\right)-\sum_{j \neq i} \beta^{j i}\left(u_{t}^{j i}+v_{t}^{j i}\right)\right] d t+\sigma^{i} d W_{t}^{i} \text { for all } i \in\{1, \ldots, n\}
$$


where $\mathbf{P}_{t}=\left(P_{t}^{1}, \ldots, P_{t}^{n}\right)$, and $W_{t}^{i}$ are (possibly correlated) Wiener processes.

With this new formulation, similar to $\S 5.2$, it is impossible to transform the problem into a single-dimension state space (the price spread). The analysis consequently becomes intractable, and numerical methods must be used.

\section{Conclusions}

\subsection{Application to Jet-Fuel (Kerosene) Prices}

In this section, we analyze the data of jet-fuel (kerosene) spot prices in two locations: New York Harbor (NY) and Los Angeles (LA). We have taken the data from the Energy Information Administration from the U.S.Goverment, see http://www.eia.doe.gov. Our objective here is to apply our model to the trading between these two locations. Trading, as we use it, can be done in several forms. For example, one can imagine a dedicated trader that buys kerosene in one market, loads it in a tank truck (which hold from 4000 to 9000 gallons), and ships it to the other market. This is an expensive way of trading, and would yield per-gallon costs of the order of 10 USD cents. The capacity available for this would probably be very large ${ }^{8}$. Our model can be used by this trader to optimize his actions. We analyze below the outcome of the actions of the traders: the spread price process.

The series reflects the spot price in USD cents per gallon from January 1991 to November 2007. We are using prices on markets that open from Monday to Friday, although in a few cases we do not have data over the entire 5-days week, due to local holidays. As a result, when calculating the spread series, we have removed the days where information of the price of one or both locations was missing. These facts (week-ends, holidays and missing data) generate some complications in our study. In fact, when calculating auto-correlations, i.e., correlations across successive days, we have to take into account this phenomenon. In other words, although not having information of the price spread in week-ends, trading on a pipeline or other shipping systems (e.g., trucks) can occur, moving product from one location to the other. So as a result, the system has three times more capacity between Friday and Monday than during the week. Thus, we index the series using natural days, i.e., the time $t$ is indexed from Monday to Sunday.

We have calculated the spread $G_{t}=P_{t}^{N Y}-P_{t}^{L A}$. Table 1 provides the basic statistics

\footnotetext{
${ }^{8}$ One can also think of an airline that flies the NY-LA route and refuels its planes every day in both locations. By buying a bit more in the cheaper market and a bit less in the more expensive market, this airline is effectively trading between the two markets. This form of trading is also expensive: a B767-400 consumes 7400 liters per hour, weights 200 tons when fully loaded, which implies that, shipping one gallon between NY and LA consumes about 0.2 gallons, around 40 USD cents per gallon traded at 2007 kerosene prices.
} 
summary of the series.

Table 1: Basic statistics of $G_{t}=P_{t}^{N Y}-P_{t}^{L A}$

\begin{tabular}{lr}
\hline Number of observations (market days) & 4238 \\
Minimum & -53.50 \\
Maximum & 44.70 \\
First quartile & -6.17 \\
Third quartile & -0.25 \\
Mean & -3.33 \\
Median & -3.25 \\
Variance & 41.12 \\
Standard deviation & 6.42 \\
Skewness & -0.20 \\
Kurtosis & 8.28 \\
\hline
\end{tabular}

Notice that the maximum and minimum value of the spread are highly significant as well as the high variance. These facts are interesting for a player trading in these markets.

It is also interesting to examine the auto-correlation diagram, shown in Figure 7. Using the time series terminology, the figure is equivalent to the autocorrelation function (ACF) but taking in account the missing data. In other words, when calculating the autocorrelation coefficients $^{9}$ at lag $k$ the terms where $G_{t}$ and/or $G_{t+k}$ are missing are not considered. The slow exponential decay in the ACF is a feature that, for example, autoregressive models (AR) exhibit, and so does ours. In fact, if the data was modeled with a simple AR(1) process, then we would have a spread process as follows: $G_{t}-\mu=\alpha\left(G_{t-1}-\mu\right)+\sigma \epsilon_{t}$ where $\epsilon_{t}$ is a white noise, with mean zero and standard deviation one. This is in some way an alternative to Equation (2).

More importantly, we depict the spread distribution of the series. Figure (8) shows both the p.d.f. and its logarithm. In our model, see Equation (5), the slope of the logarithm of the p.d.f., $f^{\prime} / f$ is equal to $-2 \beta(u(G)+v(G)) / \sigma^{2}$. This can be calculated from the figure. In other words, we can estimate $-2 \beta(u(G)+v(G)) / \sigma^{2}$ from the data. In a sense, this provides an aggregate measure for the intensity with which the market brings an off-average spread back to the average. Interestingly, this measure can be well approximated through a piecewise constant function, as follows.

\footnotetext{
${ }^{9}$ See Chatfield 2004 for more details.
} 


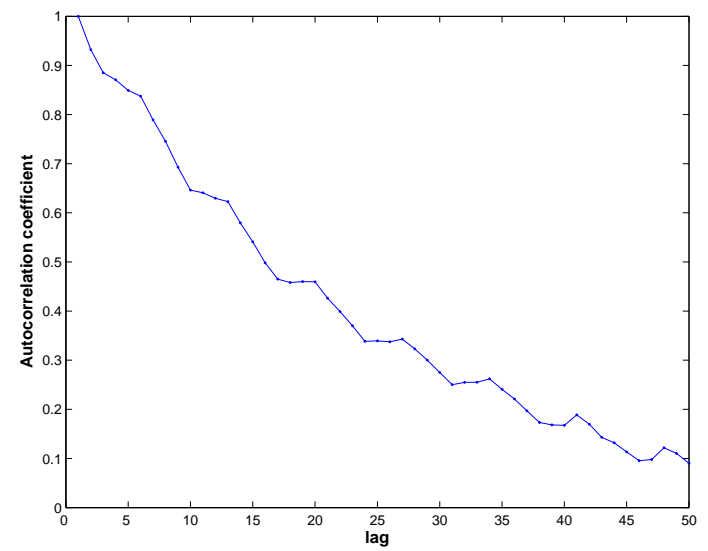

Figure 7: The autocorrelations for the jet kerosene data taking in account the missing information. The lag is measured in days.

$$
\frac{f^{\prime}}{f}(G)=-\frac{2 \beta(u(G)+v(G))}{\sigma^{2}}=\left\{\begin{aligned}
0.29 & \text { for } G \leq-5.5 \\
0.00 & \text { for }-5.5<G \leq-1.5 \\
-0.31 & \text { for } G>-1.5
\end{aligned}\right.
$$
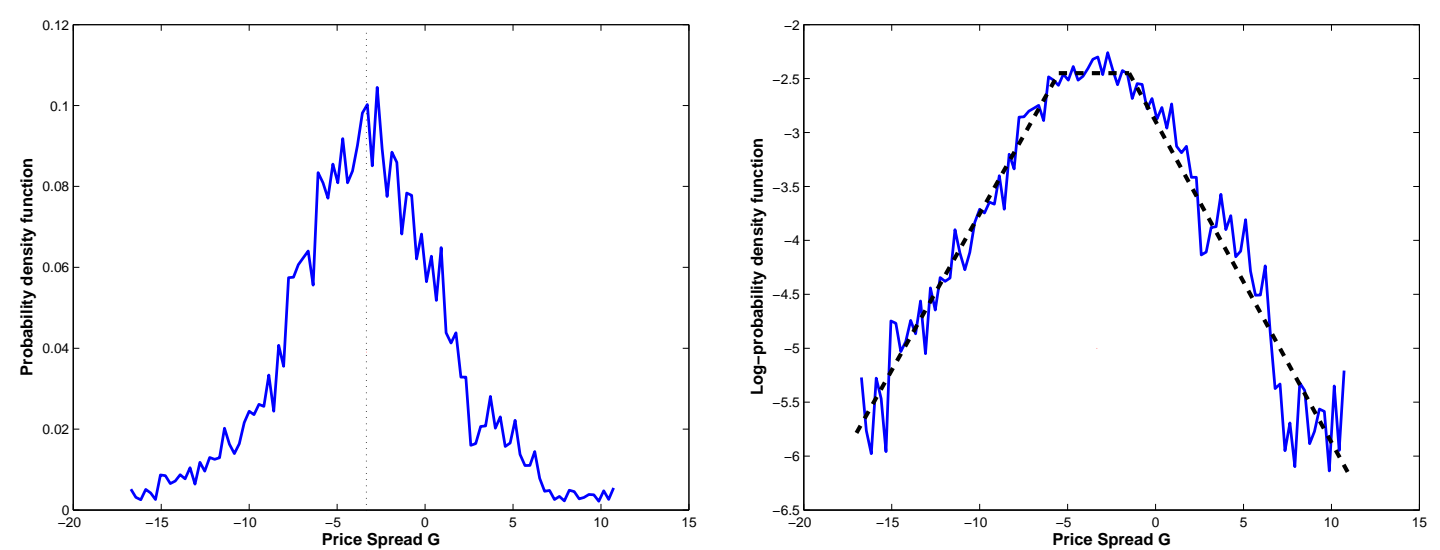

Figure 8: On the left, the shape of the p.d.f. for the data of jet kerosene is similar to what we could have expected when applying our trading policies. On the right, we show that the linear approximation (dashed line) fits the logarithm of the p.d.f. well. The vertical line reflects the mean -3.33 USD cents of the data. Finally, we plot the distribution for a data range of plus/minus two standard deviations.

The approximation fits the data well, as shown in Figure 8. Interestingly, it would be more accurate than trying to model the spread series as an $\mathrm{AR}(1)$ process. Indeed, for an $\mathrm{AR}(1)$ process, Equation (3) would yield to $\frac{f^{\prime \prime}}{f^{\prime}}=\frac{-2(1-\alpha) G}{\sigma^{2}}$. Hence the logarithm of the p.d.f. (right- 
hand side in Figure 8) would exhibit a much larger "convexity" in $G$, an approximation that does not fit the data as well as our model.

If all traders are small compared to the market (which seems a reasonable assumption), then our model would provide a way to estimate how much total trading capacity $u(G)+v(G)$ is available at each cost level $G$. This approach can be used to calibrate some of the parameters of our model. Some more detailed estimates can be obtained by directly expressing all the price variations, $\Delta G=G_{t+1}-G_{t}$, when $G_{t} \in[G-\delta, G+\delta]$, as the sum of a constant plus a white noise. This has the advantage of estimating directly $-\beta(u(G)+v(G))$ (the constant) and $\sigma$ (the standard deviation of the noise). However, it turns out that the estimates are very noisy, and only look consistent when we aggregate them significantly (large $\delta$ ), which is somehow similar to the estimation performed on $f^{\prime} / f$.

\subsection{Discussion}

The model presented in this paper analyzes the optimal trading strategy for a capacitated player between markets. We have focused on two markets only, A and B, where the prices evolve following a correlated random walk, and are influenced by the trader's actions. Under this assumption, we show that it is possible to transform the trader's decision, based on both prices $\left(P_{t}^{A}, P_{t}^{B}\right)$, into one that only depends on the price spread $G_{t}=P_{t}^{B}-P_{t}^{A}$. We characterize the optimal trading policy, which is described by two thresholds $m^{B A} \leq m^{A B}$ such that it is optimal to trade as much as possible (at capacity) from B to A when $G_{t} \leq m^{B A}$, do nothing when $m^{B A}<G_{t} \leq m^{A B}$, and again trade as much as possible, from $\mathrm{A}$ to $\mathrm{B}$ this time, when $G_{t}>m^{A B}$. This sort of policy is intuitively optimal when the trader has no impact on future prices. We show that the structure remains the same even when the trader moves the market. Interestingly, the thresholds are very close to the trading costs $c^{B A}, c^{A B}$ when the trading capacity is small, confirming that when the trader is small in the market, then he can safely assume that he has no market power (although his trading volume has an impact on the long-run distribution of prices). On the other hand, when the trading capacity is large, the thresholds can significantly deviate from the trading costs, as shown in $\S 4.4$.

In addition to the basic model, we formulate as well several extensions to more general situations, in particular where the price processes follow other dynamics, e.g., geometric Brownian motion, or the number of markets is larger than two. The trader optimization problem can there be solved numerically. These extensions can be explored further, and constitute a promising direction of future research.

Another extension of our model is to integrate the inventory aspect of the trade. We have 
considered a shipping system where the quantity bought at $\mathrm{A}$ is equal to the one sold at $\mathrm{B}$. This is a fair assumption if we focus on a pipeline. However, if the shipping system is a serial network of warehouses, then one could consider the possibility of storing inventory somewhere in that network. The analysis becomes significantly more complex.

Furthermore, we have applied the insights of our model to the analysis of the price spreads for kerosene, between New York and Los Angeles. While the average spread is of around -3 dollar cents, the deviations are fairly significant: a standard deviation of 6 dollar cents, maximum spreads of the order of half a dollar, and sometimes a positive spread for a full month. Assuming that the market is liquid, i.e., that trader enter the market as soon as the spread is higher than the transaction cost, our model can be helpful to estimate, for each level of cost, how much trading capacity is available. Such information can also be used to estimate the impact of any trading capacity addition into the price spread distribution. The specific application to kerosene spreads is just an example of what can be achieved with the model, and many alternative applications are possible, in other industries, such as LNG trade, e.g., using data from Wang et al. (2007).

Finally, we have interpreted the markets $\mathrm{A}$ and $\mathrm{B}$ as locations where an identical commodity is traded. The trading capacity represents the shipping capacity per period available, such as a pipeline, a system of trucks, etc. Interestingly, there is an alternative interpretation of our results: A and B could represent two different commodities priced in the same location. There, the trading capacity would represent the transformation capacity per period. Consider for example $P_{t}^{A}$ being the price of natural gas, and $P_{t}^{B}$ the price of electricity, in the same market. A power generation company could consider its generation capacity as trading capacity from A to B. Our model would dictate the generation policy for this company, and the distribution of the price spread (called the spark spread) could be found. Thus, our model is able to describe analytically what the trader/generator should do. In that respect, our results complement Routledge et al. (1998). 


\section{Proofs}

\section{Proposition 1}

Proof.

$\mathbb{E}\{G u(G)\}=\int_{-\infty}^{\infty} G u(G) f(G) d G=\int_{-\infty}^{\infty} G\left(-\frac{\sigma^{2}}{2 \beta} \frac{f^{\prime}(G)}{f(G)}\right) f(G) d G=\frac{\sigma^{2}}{2 \beta} \int_{-\infty}^{\infty}-G f^{\prime}(G) d G=\frac{\sigma^{2}}{2 \beta}$

after integrating by parts $G f^{\prime}(G)$ and remembering that $\int_{-\infty}^{\infty} f(G) d G=1$ as $f$ is a p.d.f.

\section{Proposition 2}

\section{Proof.}

$$
\begin{aligned}
& \mathbb{E}\left\{G u(G)-c^{A B} u^{+}(G)-c^{B A} u^{-}(G)\right\} \\
= & \int_{-\infty}^{\infty}\left(G u(G)-c^{A B} u^{+}(G)-c^{B A} u^{-}(G)\right) f(G) d G \\
= & \int_{-\infty}^{\infty}\left(G\left[-\frac{\sigma^{2}}{2 \beta} \frac{f^{\prime}(G)}{f(G)}\right]-c^{A B}\left[-\frac{\sigma^{2}}{2 \beta} \frac{f^{\prime}(G)}{f(G)}\right]^{+}-c^{B A}\left[-\frac{\sigma^{2}}{2 \beta} \frac{f^{\prime}(G)}{f(G)}\right]^{-}\right) f(G) d G \\
= & \frac{\sigma^{2}}{2 \beta}-c^{A B} \int_{-\infty}^{\infty}\left[-\frac{\sigma^{2}}{2 \beta} f^{\prime}(G)\right]^{+} d G-c^{B A} \int_{-\infty}^{\infty}\left[-\frac{\sigma^{2}}{2 \beta} f^{\prime}(G)\right]^{-} d G \\
\leq & \frac{\sigma^{2}}{2 \beta} .
\end{aligned}
$$

The profits obtained with the policy given by the proposition are:

$$
\begin{aligned}
& \int_{-\infty}^{\infty} G u(G) f(G) d G-c^{A B} \int_{M}^{\infty} q^{A B} f(G) d G-c^{B A} \int_{-\infty}^{-M} q^{B A} f(G) d G \\
= & \frac{\sigma^{2}}{2 \beta}-c^{A B} q^{A B} \int_{M}^{\infty} f(G) d G-c^{B A} q^{B A} \int_{-\infty}^{-M} f(G) d G .
\end{aligned}
$$

Now, at the limit for $M \rightarrow \infty$, this value is $\frac{\sigma^{2}}{2 \beta}$. Indeed, we have $f(G)=f(-M) e^{\frac{2 \beta q^{B A}(G+M)}{\sigma^{2}}}$ for $G<-M, f(G)=f(-M)=f(M)$ for $-M<G<M$ and $f(G)=f(M) e^{\frac{-2 \beta q^{A B}(G-M)}{\sigma^{2}}}$ for $G>M$. Of course, $f(-M)=f(M)$ is a constant such that $f$ integrates to one. As a result, $\lim _{M \rightarrow \infty} \int_{M}^{\infty} f=\lim _{M \rightarrow \infty} \int_{-\infty}^{-M} f=0$. This implies that, regardless of the policy $u(G)$, the maximum long term profit we can possibly obtain is $\frac{\sigma^{2}}{2 \beta}$. This is achieved as the limit of the proposed policy. 


\section{Theorem 1}

In order to prove Theorem 1, we first need to prove the following lemma. Proposition 3 is proved later using the optimality conditions of the theorem.

Lemma 1 Given functions $v(G)$ and $h(G):=-G v(G)$, and an optimal solution $f(G)$ of the maximization problem (11)

If there is $\left[g_{0}, g_{0}+\delta\right]$ such that $\frac{-\sigma^{2}}{2 \beta} \frac{f^{\prime}}{f}>-q^{B A}+v(G)$ then we must have

$$
\leq \frac{\int_{g_{0}}^{\infty}\left(h-c^{A B}\left[\frac{-\sigma^{2}}{2 \beta} \frac{f^{\prime}}{f}-v\right]^{+}-c^{B A}\left[\frac{-\sigma^{2}}{2 \beta} \frac{f^{\prime}}{f}-v\right]^{-}\right) f+\frac{\sigma^{2}}{2 \beta} f\left(g_{0}\right)\left(c^{A B} \mathbf{1}\left(\frac{-\sigma^{2}}{2 \beta} \frac{f^{\prime}}{f}-v \geq 0\right)-c^{B A}\left[\frac{-\sigma^{2}}{2 \beta} \frac{f^{\prime}}{f}-v\right]^{+}-c^{B A}\left[\frac{-\sigma^{2}}{2 \beta} \frac{f^{\prime}}{f}-v\right]^{-}\right) f}{\int_{-\infty}^{\infty} f} .
$$

If there is $\left[g_{0}, g_{0}+\delta\right]$ such that $\frac{-\sigma^{2}}{2 \beta} \frac{f^{\prime}}{f}<q^{A B}+v(G)$ then we must have

$$
\begin{aligned}
& \frac{\int_{g_{0}}^{\infty}\left(h-c^{A B}\left[\frac{-\sigma^{2}}{2 \beta} \frac{f^{\prime}}{f}-v\right]^{+}-c^{B A}\left[\frac{-\sigma^{2}}{2 \beta} \frac{f^{\prime}}{f}-v\right]^{-}\right) f+\frac{\sigma^{2}}{2 \beta} f\left(g_{0}\right)\left(c^{A B} \mathbf{1}\left(\frac{-\sigma^{2}}{2 \beta} \frac{f^{\prime}}{f}-v \geq 0\right)\right.}{\int^{\infty} f\left(c^{B A} \mathbf{1}\left(\frac{-\sigma^{2}}{2 \beta} \frac{f^{\prime}}{f}-v \leq 0\right)\right)} \\
& \geq \frac{\int_{-\infty}^{\infty}\left(h-c^{A B}\left[\frac{-\sigma^{2}}{2 \beta} \frac{f^{\prime}}{f}-v\right]^{+}-c^{B A}\left[\frac{-\sigma^{2}}{2 \beta} \frac{f^{\prime}}{f}-v\right]^{-}\right) f}{\int_{-\infty}^{\infty} f} .
\end{aligned}
$$

Proof. We consider an optimal $f$ that satisfies (11) such that, within an interval $\left[g_{0}, g_{0}+\delta\right]$, $\frac{-\sigma^{2}}{2 \beta} \frac{f^{\prime}}{f}>-q^{B A}+v(G)$, or in other words, $\frac{\sigma^{2}}{2 \beta} \frac{f^{\prime}}{f}<q^{B A}-v(G)$. Define $\tilde{u}(G)=u(G)+\epsilon$ for $G \in\left[g_{0}, g_{0}+\delta\right]$, and equal to $u(G)$ otherwise. The corresponding $\tilde{f}$ satisfies

$$
\frac{\tilde{f}^{\prime}(G)}{\tilde{f}(G)}= \begin{cases}\frac{f^{\prime}}{f}+\epsilon & \text { if } G \in\left[g_{0}, g_{0}+\delta\right] \\ \frac{f^{\prime}}{f} & \text { otherwise }\end{cases}
$$

and hence

$$
\tilde{f}(G)= \begin{cases}\frac{f}{\int_{-\infty}^{g_{0}} f+\int_{g_{0}}^{g_{0}+\delta} e^{\epsilon\left(G-g_{0}\right)} f(G) d G+e^{\epsilon \delta} \int_{g_{0}+\delta}^{\infty} f} & \text { if } G<g_{0} \\ \frac{f e^{\epsilon\left(G-g_{0}\right)}}{\int_{-\infty}^{g_{0}} f+\int_{g_{0}}^{g_{0}+\delta} e^{\epsilon\left(G-g_{0}\right)} f(G) d G+e^{\epsilon \delta} \int_{g_{0}+\delta}^{\infty} f} & \text { if } G \in\left[g_{0}, g_{0}+\delta\right] \\ \frac{f e^{\delta \epsilon}}{\int_{-\infty}^{g_{0}} f+\int_{g_{0}}^{g_{0}+\delta} e^{\epsilon\left(G-g_{0}\right)} f(G) d G+e^{\epsilon \delta} \int_{g_{0}+\delta}^{\infty} f} & \text { if } G>g_{0}+\delta\end{cases}
$$


In $G \in\left[g_{0}, g_{0}+\delta\right]$, for small $\epsilon>0$,

$$
\left[\frac{-\sigma^{2}}{2 \beta} \frac{\tilde{f}^{\prime}(G)}{\tilde{f}(G)}-v(G)\right]^{+}=\left[\frac{-\sigma^{2}}{2 \beta} \frac{f^{\prime}(G)}{f(G)}-v(G)\right]^{+}-\frac{\sigma^{2} \epsilon}{2 \beta} \mathbf{1}_{\left(\frac{-\sigma^{2}}{2 \beta} \frac{f^{\prime}(G)}{f(G)}-v(G) \geq 0\right)}
$$

and

$$
\left[\frac{-\sigma^{2}}{2 \beta} \frac{\tilde{f}^{\prime}(G)}{\tilde{f}(G)}-v(G)\right]^{-}=\left[\frac{-\sigma^{2}}{2 \beta} \frac{f^{\prime}(G)}{f(G)}-v(G)\right]^{-}+\frac{\sigma^{2} \epsilon}{2 \beta} \mathbf{1}_{\left(\frac{-\sigma^{2}}{2 \beta} \frac{f^{\prime}(G)}{f(G)}-v(G) \leq 0\right)}
$$

where $\mathbf{1}_{Z}=1$ if condition $Z$ is satisfied and 0 otherwise. The objective using policy $\tilde{u}$ can be written as

$$
\begin{aligned}
J_{\epsilon, \delta}(G) & =\int_{-\infty}^{\infty}\left(h-c^{A B}\left[\frac{-\sigma^{2}}{2 \beta} \frac{\tilde{f}^{\prime}(G)}{\tilde{f}(G)}-v(G)\right]^{+}-c^{B A}\left[\frac{-\sigma^{2}}{2 \beta} \frac{\tilde{f}^{\prime}(G)}{\tilde{f}(G)}-v(G)\right]^{-}\right) \tilde{f}(G) d G \\
& =\frac{\int_{-\infty}^{g_{0}} H f+e^{\epsilon \delta} \int_{g_{0}}^{\infty} H f+\int_{g_{0}}^{g_{0}+\delta} \frac{\sigma^{2} \epsilon}{2 \beta}\left(c^{A B} \mathbf{1}\left(\frac{-\sigma^{2}}{2 \beta} \frac{f^{\prime}}{f}-v \geq 0\right)-c^{B A} \mathbf{1}\left(\frac{-\sigma^{2}}{2 \beta} \frac{f^{\prime}}{f}-v \leq 0\right)\right) f e^{\epsilon\left(G-g_{0}\right)} d G}{\int_{-\infty}^{g_{0}} f+\int_{g_{0}}^{g_{0}+\delta} e^{\epsilon\left(G-g_{0}\right)} f d G+e^{\epsilon \delta} \int_{g_{0}+\delta}^{\infty} f}
\end{aligned}
$$

where $H=\left(h-c^{A B}\left[\frac{-\sigma^{2}}{2 \beta} \frac{f^{\prime}}{f}-v\right]^{+}-c^{B A}\left[\frac{-\sigma^{2}}{2 \beta} \frac{f^{\prime}}{f}-v\right]^{-}\right)$to simplify notation.

A Taylor approximation with respect to $\epsilon$ and $\delta$ (hence considering that $\delta^{2} \ll \delta$ ), yields that

$J_{\epsilon, \delta}(G)=\frac{\int_{-\infty}^{\infty} H f+\epsilon \delta\left\{\int_{g_{0}}^{\infty} H f+\frac{\sigma^{2}}{2 \beta} f\left(g_{0}\right)\left(c^{A B} \mathbf{1}_{\left(\frac{-\sigma^{2}}{2 \beta} \frac{f^{\prime}}{f}-v \geq 0\right)}-c^{B A} \mathbf{1}_{\left(\frac{-\sigma^{2}}{2 \beta} \frac{f^{\prime}}{f}-v \leq 0\right)}\right)\right\}}{\int_{-\infty}^{\infty} f+\epsilon \delta \int_{g_{0}}^{\infty} f}+o(\epsilon \delta)$

where $\lim _{x \rightarrow 0} \frac{o(x)}{x}=0$.

Since $f$ is optimal, we have $J_{\epsilon, \delta} \leq J$, for $\epsilon, \delta$ small, which yields after some algebra, that

$$
\frac{\int_{g_{0}}^{\infty} H f+\frac{\sigma^{2}}{2 \beta} f\left(g_{0}\right)\left(c^{A B} \mathbf{1}_{\left(\frac{-\sigma^{2}}{2 \beta} \frac{f^{\prime}}{f}-v \geq 0\right)}-c^{B A} \mathbf{1}_{\left(\frac{-\sigma^{2}}{2 \beta} \frac{f^{\prime}}{f}-v \leq 0\right)}\right)}{\int_{g_{0}}^{\infty} f} \leq \frac{\int_{-\infty}^{\infty} H f}{\int_{-\infty}^{\infty} f} .
$$

The same argument applied with $\frac{-\sigma^{2}}{2 \beta} \frac{f^{\prime}}{f}<q^{A B}+v(G)$ yields the second part of the lemma.

We are now ready to show the theorem.

Proof. Denote $W$ the right-hand side of the expression in Lemma 1, i.e.,

$$
W:=\frac{\int_{-\infty}^{\infty}\left(h-c^{B A}\left[-\frac{\sigma^{2}}{2 \beta} \frac{f^{\prime}}{f}-v\right]^{-}-c^{A B}\left[-\frac{\sigma^{2}}{2 \beta} \frac{f^{\prime}}{f}-v\right]^{+}\right) f d G}{\int_{-\infty}^{\infty} f}<0,
$$


from assumption (ii) of the Theorem. Consider $f$ the optimal solution of problem (11), and $u=-\frac{\sigma^{2}}{2 \beta} \frac{f^{\prime}}{f}-v$. We claim that

(i) $u(G)=q^{A B}$ when $G \rightarrow \infty$;

(ii) $u(G)=q^{A B}$ for $G>m^{A B}$ for some $m^{A B}$;

(iii) $u(G)=0$ for $m^{B A}<G<m^{A B}$ for some $m^{B A} \leq m^{A B}$; and

(iv) $u(G)=-q^{B A}$ for $G<m^{B A}$.

We start with (i). If $g_{0} \rightarrow \infty$ and $u(G)<q^{A B}$, Lemma 1 provides a necessary condition,

$$
\frac{\int_{g_{0}}^{\infty}\left(h-c^{A B}\left[\frac{-\sigma^{2}}{2 \beta} \frac{f^{\prime}}{f}-v\right]^{+}-c^{B A}\left[\frac{-\sigma^{2}}{2 \beta} \frac{f^{\prime}}{f}-v\right]^{-}\right) f+\frac{\sigma^{2}}{2 \beta} f\left(g_{0}\right)\left(c^{A B} \mathbf{1}\left(\frac{-\sigma^{2}}{2 \beta} \frac{f^{\prime}}{f}-v \geq 0\right){ }^{-c^{B A}}\left(\frac{-\sigma^{2}}{2 \beta} \frac{f^{\prime}}{f}-v \leq 0\right)\right)}{\int_{g_{0}}^{\infty} f} \geq W .
$$

The left-hand side expression of the necessary condition is bounded from above by

$$
\begin{aligned}
\frac{\int_{g_{0}}^{\infty} h f+\frac{\sigma^{2}}{2 \beta} c^{A B} f\left(g_{0}\right)}{\int_{g_{0}}^{\infty} f} & =\frac{\int_{g_{0}}^{\infty}\left(-G v(G)-\frac{\sigma^{2}}{2 \beta} c^{A B} \frac{f^{\prime}(G)}{f(G)}\right) f(G) d G}{\int_{g_{0}}^{\infty} f} \\
& \leq \frac{\int_{g_{0}}^{\infty}\left(-G v(G)+c^{A B}\left(q^{A B}+v(G)\right)\right) f(G) d G}{\int_{g_{0}}^{\infty} f} \\
& =c^{A B} q^{A B}+\frac{\int_{g_{0}}^{\infty}-\left(G-c^{A B}\right) v(G) f(G) d G}{\int_{g_{0}}^{\infty} f}
\end{aligned}
$$

Since, for $g_{0}>c^{A B},-\left(G-c^{A B}\right) v(G)$ is decreasing (because $v(G)$ is positive when $G$ is very large), the right-hand side tends to $-\infty$ as $g_{0} \rightarrow \infty$. Hence this contradicts the necessary condition from the lemma. As a result, for $G$ large enough $u(G)=q^{A B}$, and that implies (i).

Given (i), $u(G)=q^{A B}>0$ for large $G$ implies for large $g_{0}$,

$$
\begin{aligned}
& \frac{\int_{g_{0}}^{\infty}\left(h-c^{A B}\left[\frac{-\sigma^{2}}{2 \beta} \frac{f^{\prime}}{f}-v\right]^{+}-c^{B A}\left[\frac{-\sigma^{2}}{2 \beta} \frac{f^{\prime}}{f}-v\right]^{-}\right) f+\frac{\sigma^{2}}{2 \beta} f\left(g_{0}\right)\left(c^{A B} \mathbf{1}\left(\frac{-\sigma^{2}}{2 \beta} \frac{f^{\prime}}{f}-v \geq 0\right)-c^{B A} \mathbf{1}\left(\frac{-\sigma^{2}}{2 \beta} \frac{f^{\prime}}{f}-v \leq 0\right)\right)}{\int_{g_{0}}^{\infty} f} \\
= & \frac{\int_{g_{0}}^{\infty}\left(-G v(G)-c^{A B} q^{A B}\right) f(G) d G+\frac{\sigma^{2}}{2 \beta} c^{A B} f\left(g_{0}\right)}{\int_{g_{0}}^{\infty} f} \\
= & \frac{\int_{g_{0}}^{\infty}-\left(G-c^{A B}\right) v(G) f(G) d G}{\int_{g_{0}}^{\infty} f}
\end{aligned}
$$

where again we transformed $f\left(g_{0}\right)$ into $\int_{g_{0}}^{\infty}\left(-f^{\prime} / f\right) \times f$, and used Equation (5). As before, this is continuous and hence it is optimal to set $u\left(g_{0}\right)=q^{A B}$ for $g_{0}>m^{A B}$, where $m^{A B}$ is such that

$$
\frac{\int_{m^{A B}}^{\infty}\left(-G v(G)-c^{A B} q^{A B}\right) f(G) d G+\frac{\sigma^{2}}{2 \beta} c^{A B} f\left(m^{A B}\right)}{\int_{m^{A B}}^{\infty} f}=\frac{\int_{m^{A B}}^{\infty}-\left(G-c^{A B}\right) v(G) f(G) d G}{\int_{m^{A B}}^{\infty} f}=W
$$


This proves (ii). For $g_{0}=m^{A B}-\epsilon$, we have that

$$
\frac{\int_{g_{0}}^{\infty}\left(h-c^{A B}\left[\frac{-\sigma^{2}}{2 \beta} \frac{f^{\prime}}{f}-v\right]^{+}-c^{B A}\left[\frac{-\sigma^{2}}{2 \beta} \frac{f^{\prime}}{f}-v\right]^{-}\right) f+\frac{\sigma^{2}}{2 \beta} c^{A B} f\left(g_{0}\right)}{\int_{g_{0}}^{\infty} f}=W+\epsilon^{\prime}>W
$$

for $\epsilon^{\prime}>0$ very small. Is is clear that $u\left(g_{0}\right)>0$ cannot be optimal, as the necessary condition for $u<q^{A B}$ is not satisfied. Similarly, $u\left(g_{0}\right)<0$ cannot be optimal, since

$$
\frac{\int_{g_{0}}^{\infty}\left(h-c^{A B}\left[\frac{-\sigma^{2}}{2 \beta} \frac{f^{\prime}}{f}-v\right]^{+}-c^{B A}\left[\frac{-\sigma^{2}}{2 \beta} \frac{f^{\prime}}{f}-v\right]^{-}\right) f-\frac{\sigma^{2}}{2 \beta} c^{B A} f\left(g_{0}\right)}{\int_{g_{0}}^{\infty} f}=W+\epsilon^{\prime}-2 \frac{\sigma^{2}}{2 \beta} c^{B A} \frac{f\left(g_{0}\right)}{\int_{g_{0}}^{\infty} f}<W .
$$

Hence, it is optimal to set $u\left(g_{0}\right)=0$. This is true while

$$
\begin{aligned}
W & >\frac{\int_{g_{0}}^{m^{A B}}-G v(G) f(G) d G+\int_{m^{A B}}^{\infty}\left(-G v(G)-c^{A B} q^{A B}\right) f(G) d G-\frac{\sigma^{2}}{2 \beta} c^{B A} f\left(g_{0}\right)}{\int_{g_{0}}^{\infty} f} \\
& =\frac{\int_{g_{0}}^{\infty}-\left(G+c^{B A}\right) v(G) f(G) d G+\int_{m^{A B}}^{\infty}-\left(c^{A B}+c^{B A}\right) q^{A B} f(G) d G}{\int_{g_{0}}^{\infty} f}
\end{aligned}
$$

and

$$
\begin{aligned}
W & <\frac{\int_{g_{0}}^{m^{A B}}-G v(G) f(G) d G+\int_{m^{A B}}^{\infty}\left(-G v(G)-c^{A B} q^{A B}\right) f(G) d G+\frac{\sigma^{2}}{2 \beta} c^{A B} f\left(g_{0}\right)}{\int_{g_{0}}^{\infty} f} \\
& =\frac{\int_{g_{0}}^{\infty}-\left(G-c^{A B}\right) v(G) f(G) d G}{\int_{g_{0}}^{\infty} f} .
\end{aligned}
$$

These equations hold until the first one becomes an equality (since the right-hand side of the second equation is decreasing if equal to $W$, and the right-hand side of the first equation also decreases). As a result, they are satisfied for $g_{0}>m^{B A}$, which proves (iii). $m^{B A}$ is uniquely defined by

$$
\frac{\int_{m^{B A}}^{m^{A B}}-G v(G) f(G) d G+\int_{m^{A B}}^{\infty}\left(-G v(G)-c^{A B} q^{A B}\right) f(G) d G-\frac{\sigma^{2}}{2 \beta} c^{B A} f\left(m^{B A}\right)}{\int_{m^{B A}}^{\infty} f}=W .
$$

Finally, for $G<m^{B A}$, the optimal decision is to set $u(G)=-q^{B A}$, as the necessary condition for $u<-q^{B A}$ is not satisfied.

\section{Proposition 3}

Proof. Consider $m^{B A}$ and $m^{A B}$ defined in the previous proof, when $c^{A B}=c^{B A}=0$. Clearly, the case of part (iii) is now empty, and hence $m^{B A}=m^{A B}$. 


\section{Proposition 4}

Proof. $W$ is the value of the trader's problem for the optimal policy described at Theorem 1 , and the equation for $m^{A B}$ is Equation (15), given in the proof of Theorem 1. In that same proof, $m^{B A}$ is defined by

$$
\frac{\int_{m^{B A}}^{m^{A B}}-G v(G) \varphi(G) d G+\int_{m^{A B}}^{\infty}\left(-G v-c^{A B} q^{A B}\right) \varphi(G) d G-\frac{\sigma^{2}}{2 \beta} c^{B A} \varphi\left(m^{B A}\right)}{\int_{m^{B A}}^{\infty} \varphi}=W .
$$

Now taking in account the expression for $W$, the following algebra yields Equation (14).

$$
\begin{aligned}
& \left(\int_{-\infty}^{\infty} \varphi\right)\left(W-\frac{\int_{-\infty}^{m^{B A}}\left(-G v-c^{B A} q^{B A}\right) \varphi}{\int_{-\infty}^{\infty} \varphi}\right)-\frac{\sigma^{2}}{2 \beta} c^{B A} \varphi\left(m^{B A}\right)=\left(\int_{m^{B A}}^{\infty} \varphi\right) W \\
\Rightarrow & \left(\int_{-\infty}^{\infty} \varphi-\int_{m^{B A}}^{\infty} \varphi\right) W-\int_{-\infty}^{m^{B A}}\left(-G v-c^{B A} q^{B A}\right) \varphi-\frac{\sigma^{2}}{2 \beta} c^{B A} \varphi\left(m^{B A}\right)=0 \\
\Rightarrow & \frac{\int_{-\infty}^{m^{B A}}\left(-G v-c^{B A} q^{B A}\right) \varphi d G+\frac{\sigma^{2}}{2 \beta} c^{B A} \varphi\left(m^{B A}\right)}{\int_{-\infty}^{m^{B A}} \varphi}=W .
\end{aligned}
$$

Rewriting $\varphi\left(m^{B A}\right)$ as $\int_{-\infty}^{m^{B A}}\left(f^{\prime} / f\right) \times f$, and using Equation (5) yields

$\frac{\int_{-\infty}^{m^{B A}}\left(-G v-c^{B A} q^{B A}\right) \varphi d G+\frac{\sigma^{2}}{2 \beta} c^{B A} \varphi\left(m^{B A}\right)}{\int_{-\infty}^{m^{B A}} \varphi}=\frac{\int_{-\infty}^{m^{B A}}\left(-G v-c^{B A} q^{B A}-c^{B A}\left(-q^{B A}+v\right)\right) \varphi d G}{\int_{-\infty}^{m^{B A}} \varphi}$

and hence Equation (14).

\section{Proposition 5}

Proof. We denote $c^{A B}=c^{B A}=c, q^{A B}=q^{B A}=q$. Let us consider the following change of variables: $G=-s, \tilde{m}^{B A}=-m^{A B}, \tilde{m}^{A B}=-m^{B A}$ and $\tilde{\varphi}(s)=\varphi(-s)$ where $m^{B A}$ and $m^{A B}$ are given by Equations (14) and (15) in Proposition 4. We claim that if $v(-G)=-v(G)$ then $\tilde{m}^{B A}, \tilde{m}^{A B}$ and $\tilde{W}=W$ also solve the optimality equations of Proposition 4.

Indeed, we have that

$$
\begin{aligned}
\tilde{W} & =\frac{\int_{-\infty}^{\tilde{m}^{B A}}(-G v(G)-c q) \tilde{\varphi}(G) d G+\int_{\tilde{m}^{B A}}^{\tilde{m}^{A B}}-G v(G) \tilde{\varphi}(G) d G+\int_{\tilde{m}^{A B}}^{\infty}(-G v(G)-c q) \tilde{\varphi}(G) d G}{\int_{-\infty}^{\infty} \tilde{\varphi}(G) d G} \\
& =\frac{\int_{+\infty}^{-\tilde{m}^{B A}}(s v(-s)-c q) \tilde{\varphi}(-s)(-d s)+\int_{-\tilde{m}^{B A}}^{-\tilde{m}^{A B}} s v(-s)(-d s) \tilde{\varphi}(-s)(-d s)+\int_{-\tilde{m}^{A B}}^{-\infty}(s v(-s)-c q) \tilde{\varphi}(-s)(-}{\int_{+\infty}^{-\infty} \tilde{\varphi}(-s)(-d s)} \\
& =\frac{\int_{m^{A B}}^{\infty}(-s v(s)-c q) \varphi(s) d s+\int_{m^{B A}}^{m^{A B}}-s v(s) d s \varphi(s) d s+\int_{-\infty}^{m^{B A}}(-s v(s)-c q) \varphi(s) d s}{\int_{-\infty}^{\infty} \varphi(s) d s} \\
& =W
\end{aligned}
$$


where we made the change of variables $s=-G$ and used the fact that $v$ is anti-symmetrical. It is now clear that applying the change of variables,

$$
\frac{\int_{-\infty}^{\tilde{m}^{B A}}-(G+c) v(G) \tilde{\varphi}(G) d G}{\int_{-\infty}^{\tilde{m}^{B A}} \tilde{\varphi}(G) d G}=\frac{\int_{m^{A B}}^{\infty}-(G-c) v(G) \varphi(G) d G}{\int_{m^{A B}}^{\infty} \varphi(G) d G}=W=\tilde{W}
$$

i.e., satisfies Equation (14). Similarly, it satisfies Equation (15) as well. In conclusion, $\tilde{m}^{B A}, \tilde{m}^{A B}$ and $\tilde{W}$ are also solutions of the optimality equations and hence, since the solutions are unique, $m^{B A}=-m^{A B}$.

\section{Proposition 6}

Proof. When $q^{A B}=q^{B A}=0$, then $\frac{\sigma^{2}}{2 \beta} \frac{\varphi^{\prime}}{\varphi}=-v$ and hence

$$
W=\frac{\int_{-\infty}^{\infty}-G v \varphi d G}{\int_{-\infty}^{\infty} \varphi d G}=\frac{\int_{-\infty}^{\infty} G\left(\frac{\sigma^{2}}{2 \beta} \frac{\varphi^{\prime}}{\varphi}\right) \varphi d G}{\int_{-\infty}^{\infty} \varphi d G}=-\frac{\sigma^{2}}{2 \beta}
$$

On the other hand, (14) is now

$$
\begin{aligned}
W & =\frac{\int_{-\infty}^{m^{B A}}-\left(G+c^{B A}\right) v(G) \varphi(G) d G}{\int_{-\infty}^{m^{B A}} \varphi d G} \\
& =\frac{\int_{-\infty}^{m^{B A}}\left(G+c^{B A}\right) \frac{\sigma^{2}}{2 \beta} \varphi^{\prime}(G) d G}{\int_{-\infty}^{m^{B A}} \varphi d G} \\
& =\frac{\frac{\sigma^{2}}{2 \beta}\left[\left(G+c^{B A}\right) \varphi\right]_{-\infty}^{m^{B A}}-\frac{\sigma^{2}}{2 \beta} \int_{-\infty}^{m^{B A}} \varphi}{\int_{-\infty}^{m^{B A}} \varphi d G} \\
& =-\frac{\sigma^{2}}{2 \beta}+\frac{\sigma^{2}}{2 \beta} \frac{\left(c^{B A}+m^{B A}\right) \varphi\left(m^{B A}\right)}{\int_{-\infty}^{m^{B A}} \varphi}
\end{aligned}
$$

Thus as the optimal solution is unique, we have that $m^{B A}+c^{B A}=0$ and hence $m^{B A}=-c^{B A}$. Similarly $m^{A B}=c^{A B}$ when $q^{A B}=q^{B A}=0$.

When $q^{A B}, q^{B A}>0$ but very small, we know that $\left(m^{B A}, m^{A B}\right)$ are uniquely defined by

$$
\begin{aligned}
& 0=\frac{\int_{m^{A B}}^{\infty}-\left(G-c^{A B}\right) v(G) \varphi(G) d G}{\int_{m^{A B}}^{\infty} \varphi} \\
& -\frac{\int_{-\infty}^{m^{B A}}\left(-G v(G)-c^{B A} q^{B A}\right) \varphi(G) d G+\int_{m B A}^{m^{A B}}-G v(G) \varphi(G) d G+\int_{m}^{\infty}{ }^{\infty}\left(-G v(G)-c^{A B} q^{A B}\right) \varphi(G) d G}{\int_{-\infty}^{\infty} \varphi d G} \\
& 0=\frac{\int_{-\infty}^{m^{B A}}-\left(G+c^{B A}\right) v(G) \varphi(G) d G}{\int_{-\infty}^{m^{B A}} \varphi} \\
& -\frac{\int_{-\infty}^{m^{B A}}\left(-G v(G)-c^{B A} q^{B A}\right) \varphi(G) d G+\int_{m B A}^{m^{A B}}-G v(G) \varphi(G) d G+\int_{m}^{\infty}{ }^{\infty}\left(-G v(G)-c^{A B} q^{A B}\right) \varphi(G) d G}{\int_{-\infty}^{\infty} \varphi d G} .
\end{aligned}
$$


This is a system of two equations $\psi_{1}\left(m^{B A}, m^{A B}, q^{B A}, q^{A B}\right)=0$ and $\psi_{2}\left(m^{B A}, m^{A B}, q^{B A}, q^{A B}\right)=$ 0 with two unknowns, and two parameters $q^{B A}, q^{A B}$. Since $\psi$ is continuously differentiable, then $\left(m^{B A}, m^{A B}\right)$ is continuous in $\left(q^{B A}, q^{A B}\right)$ around $(0,0)$. We thus have that, for small $q^{B A}, q^{A B}$, the deviations around $m^{B A}=-c^{B A}$ and $m^{A B}=c^{A B}$ are respectively $m^{B A}+c^{B A}$ and $m^{A B}-c^{A B}$, and hence

$$
\frac{d \psi}{d q^{B A}} q^{B A}+\frac{d \psi}{d q^{A B}} q^{A B}+\frac{d \psi}{d m^{B A}}\left(m^{B A}+c^{B A}\right)+\frac{d \psi}{d m^{A B}}\left(m^{A B}-c^{A B}\right)+o\left(q^{B A}\right)+o\left(q^{A B}\right)=0,
$$

where $o(x) / x \rightarrow 0$ when $x \rightarrow 0$.

Interestingly, at $q^{A B}=0$,

$$
\frac{1}{\varphi} \frac{\partial \varphi}{\partial m^{B A}}=\frac{2 \beta}{\sigma^{2}} v\left(-c^{B A}\right) \text { and } \frac{1}{\varphi} \frac{\partial \varphi}{\partial m^{A B}}=0 .
$$

Also,

$$
\frac{1}{\varphi} \frac{\partial \varphi}{\partial q^{B A}}=-\frac{2 \beta}{\sigma^{2}}\left(-c^{B A}-G\right)^{+} \text {and } \frac{1}{\varphi} \frac{\partial \varphi}{\partial q^{A B}}=-\frac{2 \beta}{\sigma^{2}}\left(G-c^{A B}\right)^{+} .
$$

Hence, at $q^{A B}=q^{B A}=0$, we have that

$$
\begin{gathered}
\frac{1}{\psi_{1}} \frac{d \psi_{1}}{d m^{B A}}=\frac{-\varphi\left(-c^{B A}\right)}{\int_{-\infty}^{-c^{B A}} \varphi} \text { and } \frac{1}{\psi_{1}} \frac{d \psi_{1}}{d m^{A B}}=0 \\
\frac{1}{\psi_{2}} \frac{d \psi_{2}}{d m^{A B}}=\frac{\varphi\left(c^{A B}\right)}{\int_{c^{A B}}^{\infty} \varphi} \text { and } \frac{1}{\psi_{2}} \frac{d \psi_{2}}{d m^{B A}}=0
\end{gathered}
$$

In addition, using that $W=-\frac{\sigma^{2}}{2 \beta}$,

$$
\begin{gathered}
\frac{1}{\psi_{1}} \frac{d \psi_{1}}{d q^{B A}}=\left(\frac{2 \beta}{\sigma^{2}}\right)\left[\begin{array}{l}
\frac{2 \beta}{\sigma^{2}} \frac{\int_{-\infty}^{-c^{B A}}\left(G+c^{B A}\right)^{2} v(G) \varphi(G) d G}{\int_{-\infty}^{-c^{B A}} \varphi}+\frac{\int_{-\infty}^{-c^{B A}}-\left(G+c^{B A}\right) \varphi(G) d G}{\int_{-\infty}^{-c^{B A}} \varphi} \\
-c^{B A} \frac{\int_{-\infty}^{-c^{-} A} \varphi}{\int_{-\infty}^{\infty} \varphi}-\frac{2 \beta}{\sigma^{2}} \frac{\int_{-\infty}^{-c^{B A}} G\left(G+c^{B A}\right) v(G) \varphi(G) d G}{\int_{-\infty}^{\infty} \varphi}-\frac{\int_{-\infty}^{-c^{B A}}-\left(G+c^{B A}\right) \varphi(G) d G}{\int_{-\infty}^{\infty} \varphi}
\end{array}\right], \\
\frac{1}{\psi_{1}} \frac{d \psi_{1}}{d q^{A B}}=\left(\frac{2 \beta}{\sigma^{2}}\right)\left[-c^{A B} \frac{\int_{c A B}^{\infty} \varphi}{\int_{-\infty}^{\infty} \varphi}+\frac{2 \beta}{\sigma^{2}} \frac{\int_{c A B}^{\infty} G\left(G-c^{A B}\right) v(G) \varphi(G) d G}{\int_{-\infty}^{\infty} \varphi}-\frac{\int_{c}^{\infty}{ }^{\infty}\left(G-c^{A B}\right) \varphi(G) d G}{\int_{-\infty}^{\infty} \varphi}\right]
\end{gathered}
$$

and

$$
\begin{gathered}
\frac{1}{\psi_{2}} \frac{d \psi_{2}}{d q^{B A}}=\left(\frac{2 \beta}{\sigma^{2}}\right)\left[-c^{B A} \frac{\int_{-\infty}^{-c^{B A}} \varphi}{\int_{-\infty}^{\infty} \varphi}-\frac{2 \beta}{\sigma^{2}} \frac{\int_{-\infty}^{-c^{B A}} G\left(G+c^{B A}\right) v(G) \varphi(G) d G}{\int_{-\infty}^{\infty} \varphi}-\frac{\int_{-\infty}^{-c^{B A}}-\left(G+c^{B A}\right) \varphi(G) d G}{\int_{-\infty}^{\infty} \varphi}\right] \\
\frac{1}{\psi_{2}} \frac{d \psi_{2}}{d q^{A B}}=\left(\frac{2 \beta}{\sigma^{2}}\right)\left[\begin{array}{l}
-\frac{2 \beta}{\sigma^{2}} \frac{\int_{c^{A B}}^{\infty}\left(G-c^{A B}\right)^{2} v(G) \varphi(G) d G}{\int_{c^{A B}}^{\infty} \varphi}+\frac{\int_{c^{A B}}^{\infty}\left(G-c^{A B}\right) \varphi(G) d G}{\int_{c}^{\infty}{ }^{\infty} \varphi} \\
-c^{A B} \frac{\int_{c A B}^{\infty} \varphi}{\int_{-\infty}^{\infty} \varphi}+\frac{2 \beta}{\sigma^{2}} \frac{\int_{c^{A B}}^{\infty} G\left(G-c^{A B}\right) v(G) \varphi(G) d G}{\int_{-\infty}^{\infty} \varphi}-\frac{\int_{c^{A B}}^{\infty}\left(G-c^{A B}\right) \varphi(G) d G}{\int_{-\infty}^{\infty} \varphi}
\end{array}\right]
\end{gathered}
$$


As a result, we can express Equation (21) as

$$
m^{B A}=-c^{B A}-\left(\frac{\psi_{1}}{\frac{d \psi_{1}}{d m^{B A}}}\right)\left(\frac{1}{\psi_{1}} \frac{d \psi_{1}}{d q^{B A}} q^{B A}+\frac{1}{\psi_{1}} \frac{d \psi_{1}}{d q^{A B}} q^{A B}\right)+o\left(q^{B A}\right)+o\left(q^{A B}\right)
$$

and

$$
m^{A B}=c^{A B}-\left(\frac{\psi_{2}}{\frac{d \psi_{2}}{d m^{A B}}}\right)\left(\frac{1}{\psi_{2}} \frac{d \psi_{2}}{d q^{B A}} q^{B A}+\frac{1}{\psi_{2}} \frac{d \psi_{2}}{d q^{A B}} q^{A B}\right)+o\left(q^{B A}\right)+o\left(q^{A B}\right) .
$$

\section{References}

[1] Bertsekas D. P. 2000. Dynamic Programming and Optimal Control. Athena Scientific, Belmont, Massachusetts.

[2] Caldentey R., R. Epstein and D. Sauré 2007. "Optimal Explotation of Nonrenewable Resource." Working paper, Stern School, New York University.

[3] Chambers M. and R. Bailey 1996. "A theory of commodity price fluctuations." The Review of Economic Studies, 104, pp. 429-441.

[4] Chatfield C. 2004. The Analysis of Time Series. An introduction. Sixth Edition. Chapman\&Hall

[5] Deaton A. and G. Laroque 1992. "On the Behaviour of Commodity Prices." The Review of Economic Studies, 59(1), pp. 1-23.

[6] Deaton A. and G. Laroque 1996. "Competitive Storage and Commodity Price Dynamics." The Journal of Political Economy Studies, 104(5), pp. 896-923.

[7] Dong L. and H. Liu 2007. "Equilibrium Forward Contracts on Nonstorable Commodities in the Presence of Market Power". Operations Research, 55(1), pp. 128-145.

[8] Gibson R. and E. Schwartz 1990. "Stochastic convenience yield and the pricing of oil contingent claims." Journal of Finance, 45, pp. 959-976.

[9] Goel A. and G.J. Gutierrez 2004. "Integrating Spot and Futures Commodity Markets in the Optimal Procurement Policy of an Assemble-to-Order Manufacturer". Working paper, Department of Information Risk and Operations Management, University of TexasAustin.

[10] Goel A. and G.J. Gutierrez 2006. "Integrating Commodity Markets in the Optimal Procurement Policies of a Stochastic Inventory System". Working paper, Department of Information Risk and Operations Management, University of Texas-Austin. 
[11] Goel A. and G.J. Gutierrez 2007. "Procurement and Distribution Policies in a Distributive Supply Chain in the Presence of Commodity Markets". Working paper, Department of Information Risk and Operations Management, University of Texas-Austin.

[12] Golabi K. 1985. "Optimal Inventory Policies when Ordering Prices are Random." Operations Research, 33(3), pp. 575-588.

[13] Hull J. 2003. Options, Futures and other Derivatives. Prentice Hall Upper Saddle River, NJ.

[14] Routledge B. R., D. J. Seppi and C. S. Spatt 1998. "The Spark Spread: An Equilibrium Model of Cross-Commodity Price Relationships in Electricity". Working paper, Graduate School of Industrial Administration, Carnegie Mellon University.

[15] Routledge B. R., D. J. Seppi and C. S. Spatt 2000. "Equilibrium Forward Curves for Commodities". The Journal of Finance, 50(3), pp. 1297-1338.

[16] Schwartz E. 1997. "The Stochastic Behaviour of Commodity Prices: Implications for Valuation and Hedging." The Journal of Finance, 52 (3), pp. 923-973.

[17] Schwartz E. and J. E. Smith 2000. "Short-Term Variations and Long-Term Dynamics in Commodity Prices" Management Science, 46 (7), pp. 893-911.

[18] Secomandi N. 2004. "Valuation of Contracts for Interstate Natural Gas Pipeline Transportation Capacity by producers and Local Distribution Companies." Working paper, Tepper School of Business, Carnegie Mellon University.

[19] Secomandi N. 2005. "Optimal Inventory-Trading Policy for Commodity Storage Assets." Working paper, Tepper School of Business, Carnegie Mellon University.

[20] Wang M. X., N. Secomandi, S. Kekre and A. Scheller-Wolf 2007. "Valuation of Downstream Liquefied-Natural-Gas Storage." Working paper, Tepper School of Business, Carnegie Mellon University.

[21] Wang Y. 2001. "The optimality of myopic stocking policies for systems with decreasing purchasing prices." European Journal of Operational Research, 133, pp.153-159

[22] Williams J. and B. Wright 1991. Storage and Commodity Markets. Cambridge Universtity Press, Cambridge, England.

[23] Wilmot P., J. Dewynne and S. Howinson 1997. Option Pricing. Oxford Financial Press.

[24] Zipkin P. H. 2000. Foundations of Inventory Management. McGraw-Hill International Editions. 